\title{
RICERCHE SU PIANO RETRIBUTIVO E RETRIBUZIONE DOCENTI: UNO STATO DELL'ARTE - 2008-2021
}

\section{ARTICOLO ORIGINALE}

LIMA, Maria José Rocha ${ }^{1}$, RAMOS, Fernando Sadio²

LIMA, Maria José Rocha. RAMOS, Fernando Sadio. Ricerche su piano retributivo

e retribuzione docenti: uno stato dell'arte - 2008-2021. Revista Científica

Multidisciplinar Núcleo do Conhecimento. Anno. 06, Ed. 11, Vol. 08, pp. 100-133.

Novembre 2021. ISSN: 2448-0959, Link di accesso:

https://www.nucleodoconhecimento.com.br/formazione-it/retribuzione-docenti, DOI:

10.32749/nucleodoconhecimento.com.br/formazione-it/retribuzione-docenti

\section{RIEPILOGO}

Questo articolo presenta un'indagine sugli studi sul piano salariale e sulla retribuzione degli insegnanti tra il 2008 e il 2021[3]. In questo senso, la revisione della letteratura sul piano salariale del magistero è un pezzo fondamentale. A tal fine, la metodologia sviluppata per la ricerca bibliografica si basa sull'uso scientifico e attento della teoria della Evidence-Based Practice $(E B P)$ - in portoghese, Práticas Baseadas em Evidências (PBE). È uno stato dell'arte che taglia fuori uno dei fattori più decisivi per la trasformazione della realtà educativa: gli studi sul piano salariale e la retribuzione degli insegnanti brasiliani. In questo studio viene esposta un'altra contraddizione tra discorsi enfatici o magnilohot in difesa della valorizzazione

\footnotetext{
${ }^{1}$ Dottorando in Pedagogia presso l'Università Internazionale Iberoamericana - UNINI -; Master in Pedagogia dell'Università Federale di Bahia-UFBA-, Psicopedagogia dell'Università Cândido Mendes - AVM, Specialista in Psicoanalisi dell'Associazione Brasiliana di Studi e Ricerca in Psicoanalisi - ABEPP-, Specialista in Culture Nere nell'Atlantico dell'Università di Brasília - UnB-, Specialista in Metodologia dell'insegnamento dell'istruzione superiore, Facoltà di Scienze della Formazione di Bahia - FEBA - Laurea completa per la formazione speciale nel curriculum delle scuole superiori, nell'area delle scienze della salute; Insegnante elementare, diplomata all'Instituto Central de Educação Isaías Alves. ORCID: 0000-0003-1766-2169

${ }^{2}$ Consulente. Dottore in Scienze Sociali/Scienze dell'Educazione, Programma Curriculum, Profesorado e Instituciones Educativas, Università di Granada, Spagna. Master in Filosofia Contemporanea dell'Università di Coimbra, Portogallo. Laurea in Filosofia presso I'Università di Coimbra, Portogallo. Direttore di DEDICA - Rivista di educazione e scienze umane.. (0000-0001-7654-5638) - ORCID
}

RC: 101663

Disponibile in: https://www.nucleodoconhecimento.com.br/formazione-it/retribuzionedocenti 
dell'insegnamento e delle pratiche delle autorità politiche ed educative, chiamandoci anche la bassa produzione di monografie, tesi e tesi di dottorato, nel mondo accademico, sul piano salariale e sulla retribuzione degli insegnanti. Anche con la proclamazione dell'apprezzamento dell'insegnante come fattore decisivo per garantire la qualità dell'istruzione, sono passati più di dieci anni dall'istituzione del piano salariale, tuttavia, circa il $60 \%$ dei comuni brasiliani non to ha ancora implementato e questo non ha il riverbero atteso negli studi accademici.

Parole chiave: Stato dell'arte, Piano salariale, Stipendio dell'insegnante, Stipendio dell'insegnante, Professionalizzazione dell'insegnante.

\section{INTRODUZIONE}

In Brasile, purtroppo a causa di unicuique tradizione culturale, la garanzia dell'educazione come diritto sociale è stata segnata da un ritardo secolare e da una lentezza impensabile. Le cause di tale ritardo educativo sono state indicate da teorici, come il sociologo Florestan Fernandes (1989, p. 160), che analizzando la storia brasiliana identifica la dipendenza economica, l'oscurantismo delle classi dominanti e un conflitto permanente portato all'interno della scuola, che aiuta poco a risolvere i problemi, come fattori di ritardo. È come una misurazione ininterrotta dei rapporti di forza. Quindi, ci sono controversie sul progetto scolastico, sul progetto di valorizzazione, o meno, dell'insegnante; progetto di società; il progetto dell'uomo, finalmente, come una lotta politica incessante. Quello che fa un governo, un altro lo annulla. Quali sono questi interessi che muovono la mancata applicazione della legge che ha istituito il piano salariale? Sarebbero espresse le dispute di cui florestano Fernandes ci parla, tra gli scettici di destra o di sinistra che quasi sempre sabotano l'istruzione in Brasile?

Nel 1932, il Manifesto dei Pionieri della Nuova Educazione, che aveva tra i suoi abbonati più importanti Fernandes de Azevedo e Anísio Teixeira, inizia il documento che chiede che nella gerarchia dei problemi nazionali, nessuno sarebbe stato superato dall'istruzione, nemmeno quelli di carattere economico. II famoso pensatore 
dell'educazione Anísio Teixeira ha avvertito che "senza educazione, democrazia sarebbe una parola vana usata per giustificare la triste farsa di un suffragio universale derisorio" (LIMA, 2011, p. 66). Per Florestan, "un punto essenziale è ciò che riguarda la tradizione culturale e ciò che ha rappresentato della limitazione culturale dell'insegnante, meno in teoria, che in pratica (FERNANDES, 1898, p. 157).

La visione del magistero come sacerdozio si è incrociata e consolidata attraverso i secoli. Questa concezione religiosa e tradizionale nella cultura brasiliana, costruita bene nei primi giorni della colonizzazione, quando diretta da religiosi, era uno dei gravi problemi affrontati dagli insegnanti per la loro professionalizzazione e lo svolgimento del lavoro scolastico professionale. In questa tradizione era, ed è, inaccettabile per l'insegnante discutere di retribuzione; il maestro dovrebbe prendere il magistero come una missione: essere idealista, essere povero e felice nell'esercizio della professione. Non è raro che l'insegnante non abbia nemmeno i soldi per comprare libri.

Nel 1827, l'imperatore Dom Pedro I concesse la legge delle scuole di lettere del 15 ottobre 1827, stabilendo nell'articolo 3 la remunerazione degli insegnanti, un piano salariale mai attuato. II suo successore, don Pedro II, affermò addirittura che "se non fosse stato imperatore, avrebbe voluto essere un maestro" e che non conosceva "una missione più grande e più nobile di quella di dirigere la giovane intelligenza e preparare gli uomini del futuro". L'ultimo monarca dell'Impero del Brasile regnò per quasi 50 anni e nulla di concreto fece per la remunerazione e il riconoscimento dell'insegnante.

Le belle ed espressive parole pronunciate e gli atti legislativi degli imperatori del Brasile, dal 1827 al 1889, non hanno avuto le ripercussioni annunciate nella politica della remunerazione degli insegnanti. Nonostante la mancata attuazione del pavimento magisteriale, l'atto di creare il pavimento nel $1827 \mathrm{fu}$ emblematico. Tutto ciò costituisce la prova più eloquente di questa discrepanza tra discorso e pratica nella storia politica della remunerazione degli insegnanti nelle scuole pubbliche brasiliane.

RC: 101663

Disponibile in: https://www.nucleodoconhecimento.com.br/formazione-it/retribuzionedocenti 
Le autorità brasiliane parlano con eloquenza dell'importanza dell'insegnante, tuttavia,

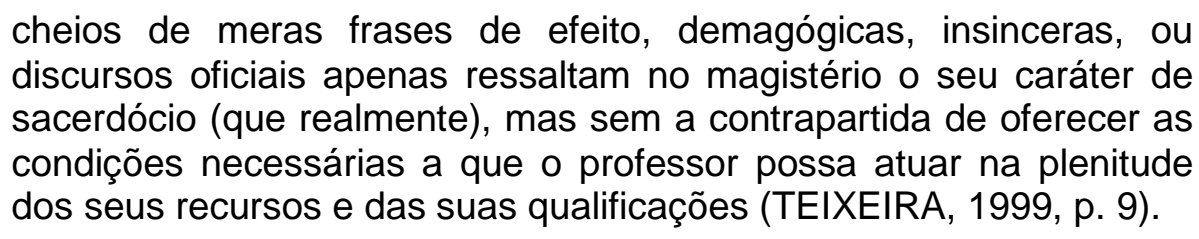

Questi discorsi, che spesso si trasmettono, dal punto di vista del magistero come missione di carattere religioso e/o assistance vista/paternalistico sembrano funzionare, di regola, impedendo agli educatori, agli accademici e persino ai sindacalisti, di affrontare e difendere gli stipendi, il piano salariale, la retribuzione decente dell'insegnamento per il magistero, in modo che quando lo fanno, è con una certa tibieza.

L'idea del "maestro vocazionale", una visione puramente soggettiva, ha un impatto negativo sul compito pedagogico, costituendo una barriera al superamento della visione errata che per insegnare, alcune abilità sono sufficienti, senza tener conto della complessità del compito. L'insegnamento non è lavoro per i laici. La didattica e la pedagogia sono campi scientifici con un significativo accumulo di conoscenze.

Pertanto, due gravi sfide devono essere affrontate per l'esecuzione del lavoro scolastico professionale e l'aumento della qualità dell'istruzione: il non riconoscimento dell'insegnamento come professione e l'incertezza circa lo scopo della scuola. Per superare questi due problemi, è necessario fare un movimento forte che si opponga alla situazione impensabile che è l'insegnante che riceve lo stipendio minimo quasi sempre ben al di sotto degli stipendi ricevuti da professionisti con lo stesso livello di formazione. Questa situazione lo costringe a lavorare in più scuole contemporaneamente e ad accettare ancora l'esecuzione del lavoro scolastico in spazi inadeguati.

Gli insegnanti lavorano nelle condizioni più precarie: accanto a bagni fetidi, in scuole con stanze divise anche da tabiques, senza acqua, senza soggiorno. Funzionano più spesso senza attrezzature di base, come portafogli, scrivanie, armadi, tra gli altri 
necessari. In luoghi lontani, inospitali e non protetti, le aggressioni e le aggressioni agli insegnanti all'interno delle scuole sono comuni.

Entro il 2020, 4.300 scuole pubbliche non avevano bagni e Internet a banda larga non ha raggiunto $17.200(20,5 \%)$ nel 2020. Inoltre, 35.800 scuole continuano senza raccolta delle acque reflue, il $26,6 \%$ del totale, secondo il censimento scolastico del 2019. Per quanto riguarda le risorse didattico-pedagogiche, gli insegnanti, perché non le hanno, competono ingiustamente con le risorse dei media, come stiamo vedendo in questo periodo di pandemia. II Covid-19 ha messo in luce la tragica situazione degli insegnanti "sputati e gesso", senza mappe, globi, illustrazioni, nel $21^{\circ}$ secolo, e nell'era della rivoluzione tecnologica.

È anche essenziale, per la risoluzione di questi problemi, la chiara definizione dello scopo della scuola. Per questo, è necessario stimolare e aumentare la produzione scientifica e un movimento che promuova il superamento di questa confusione stabilita in relazione alla funzione della scuola: luogo di protezione, rifugio, deposito per i bambini o spazio unico per lo svolgimento del lavoro scolastico, che deve adempiere al suo scopo primario di offrire le conoscenze tecniche e scientifiche e i contenuti necessari per la sopravvivenza simbolica.

Uno dei discorsi più pervasivi per la decostruzione di questa visione dell'insegnantesacerdote è stato pronunciato dal professore dell'Università di Bahia - UFBA, giornalista e scrittore João Carlos Teixeira, nell'Assemblea legislativa da Bahia, nel 1999, quando ha detto:

Por lamentável (e iníqua) tradição, no Brasil o professor costuma ser simultaneamente mártir e herói, quando deveria ser apenas (sobretudo) uma pessoa capacitada para o exercício da sua relevante missão social, prestigiada pela sociedade e amparada pelos poderes públicos, com remuneração à altura da importância de que o seu trabalho se reveste para toda a coletividade (TEIXEIRA, 1999, p. 9).

Solo nel $21^{\circ}$ secolo è stato istituito per legge il Piano salariale professionale nazionale. È così che il 16 luglio 2008, I'allora Presidente della Repubblica, Luiz 
Inácio Lula da Silva, ha sancito la legge 11.738/2008. Questa legge ha istituito il Piano salariale professionale nazionale per i professionisti dell'istruzione pubblica di base - PSPN - stabilendo un valore da, obbligatoriamente, adeguato ogni anno e "al di sotto del quale l'Unione, gli Stati, il Distretto Federale e i Comuni non saranno in grado di fissare lo stipendio iniziale delle Carriere dell'insegnamento pubblico dell'istruzione di base, per il viaggio di, massimo di 40 (quaranta) ore settimanali" (BRASIL, 2008). Tuttavia, anche dopo questa attesa secolare, più della metà dei 5.570 comuni brasiliani non si conforma.

In considerazione di questo scenario $\mathrm{e}$ in conformità con la proposta, abbiamo selezionato i dieci autori che hanno pubblicato di più sul piano salariale dell'insegnamento pubblico dell'istruzione di base e cercato di catturare i risultati più eccezionali di questi autori in relazione all'attuazione del PSPN , istituito dalla Legge 11.738, del 16 luglio 2008.

Le persone, le autorità educative, gli imprenditori e soprattutto i politici fanno discorsi eloquenti e aggettivi di riconoscimento all'insegnante, ma la pratica è palesemente contraddittoria.

\section{STIPENDIO MINIMO E RETRIBUZIONE DEGLI INSEGNANTI: UNA REVISIONE DELLA LETTERATURA}

Come precedentemente contestualizzato, l'oggetto di questo lavoro è l'implementazione del piano salariale degli insegnanti, su cui presentiamo questa revisione della letteratura. A tal fine, la metodologia sviluppata per la ricerca bibliografica si basa sull'uso scientifico e attento della teoria della Evidence-based practice (EBP).

È qui interessante notare che la discussione sulle ricerche bibliografiche basate sull'evidenza nasce dagli studi di Medicina basata sull'evidenza (MBE) e si espande ad altre discipline con il termine EBP. Secondo Santos et al. (2007), 
a PBE prevê metodologias e processos para a identificação de evidências de que um certo tratamento, ou meio diagnóstico, é efetivo, estratégias para avaliação da qualidade dos estudos e mecanismos para a implementação na assistência. (...) O movimento da PBE teve origem simultânea na McMaster University (Ontario, Canadá) e na University of York (Reino Unido). Evidência é aquilo que é claro, a constatação de uma verdade que não suscita qualquer dúvida. Evidência científica representa uma prova de que um determinado conhecimento é verdadeiro ou falso. Para que se tenha evidência científica é necessário que exista pesquisa prévia, conduzida dentro dos preceitos científicos (SANTOS et al., 2007).

In questo senso, la proposta è stata quella di effettuare una ricerca bibliografica basata su precetti scientifici con l'obiettivo di estrarre dall'oggetto principale "pagamento del Piano Nazionale di Stipendio Professionale - PSPN", il lavoro di ricerca più correlato e importante per lo studio.

Inoltre, giustifichiamo l'adozione di questa metodologia perché ci sono critiche alle recensioni delle letterature tradizionali, che non hanno metodologie specifiche ed esplicite, dimostrando così totale casualità e parzialità nelle loro ricerche. Santos et al. ( 2007) sollevare questa discussione quando espone che:

As revisões de literatura tradicionais (hoje chamadas revisões narrativas) há muito são criticadas, uma vez que o método de busca bibliográfica e seleção dos estudos não são padronizados e explicitados. Os resultados obtidos com tais revisões são tendenciosos, não esgotam toda a literatura disponível sobre o tema pesquisado e geralmente são inconclusivos. A busca de evidência requer adequada definição da pergunta de pesquisa e criação de estrutura lógica para a busca bibliográfica de evidências na literatura, que facilitam e maximizam o alcance da pesquisa (SANTOS et al., 2007).

Secondo Bariani et al. (2007), per le ricerche bibliografiche basate sull'evidenza, dovrebbero essere seguite alcune linee guida, come abbiamo fatto in questo lavoro:

I. Le ricerche di letteratura oggi sono in gran parte in directory online, cioè in database online. Per questo lavoro, abbiamo condotto una ricerca nel database di Capes, Scientific Electronic Library Online - Scielo, Google Scholar e Google Books.

RC: 101663

Disponibile in: https://www.nucleodoconhecimento.com.br/formazione-it/retribuzionedocenti 
II. Selezione di descrittori, o parole chiave, allineati con le specificità del tema. In questo modo, evitiamo ricerche con descrittori ampi e generalisti.

III. Delimitazione della tipicità delle parole chiave, dando priorità all'uso di nomi e aggettivi; uso di parole al singolare e, infine, evitando preposizioni, congiunzioni o articoli.

IV. Verifica e validazione delle parole scelte sui siti editoriali nelle principali aree di studio relative alla ricerca.

V. Filtro di ricerca. La ricerca deve essere ben delimitata, quindi ci sono alcuni modi che sono stati seguiti in questa ricerca:

A) Ricerca del materiale nell'arco temporale della ricerca;

B) Ricerca esatta dei termini. La ricerca esatta è facilitata quando parole o frasi sono scritte tra virgolette;

C) Uso di operatori logici booleani AND, OR e NOT, dove AND è usato per individuare più di un termine nello stesso riferimento; NOT escludere un termine comune dall'essere trovato con la parola chiave utilizzata; e OR per escludere termini più comuni dall'essere trovati con la parola chiave utilizzata.

Tabella 1 - Espressioni chiave di ricerca

Piano salariale AND magistero

\begin{tabular}{|l|l|}
$\begin{array}{l}\text { Stipendio minimo AND formazione } \\
\text { professionale }\end{array}$ & $\begin{array}{l}\text { PSPN } \\
\text { professionale }\end{array}$ \\
\hline $\begin{array}{l}\text { Stipendio piano AND insegnante } \\
\text { Stipendio piano AND insegnante }\end{array}$ & PSPN AND insegnante \\
\hline $\begin{array}{l}\text { Piano salariale AND educatore } \\
\text { Salario minimo AND istruzione dei } \\
\text { lavoratori }\end{array}$ & PSPN AND insegnante \\
\hline
\end{tabular}

RC: 101663

Disponibile in: https://www.nucleodoconhecimento.com.br/formazione-it/retribuzione$\underline{\text { docenti }}$ 


\section{Stipendio piano AND istruzione}

PSPN AND istruzione

Fonte: Elaborazione propria (2021).

Basandosi sul tema del Piano salariale professionale nazionale, sono stati delimitati i termini chiave, che sono Piano salariale e PSPN. Le ricerche sono state effettuate con il ritaglio dal 1 gennaio 2008 al 1 gennaio 2021 in modalità "ricerca avanzata" sul portale CAPES, Scielo, Google Academic e Google Books. I termini OR e AND sono stati quindi classificati come operatori booleani. La combinazione di termini è stata eseguita per cercare nei database i termini: Piano salariale o PSPN, combinando la ricerca con sinonimi concettuali sugli insegnanti. Inoltre, vale la pena notare che le preposizioni "di" e "di" sono state rimosse allo scopo di affinare la ricerca.

Dopo la ricerca della letteratura, è stato creato un database con tutti gli articoli, le tesi e i libri trovati. Per l'assemblaggio della banca dati, sono stati ignorati i documenti ripetuti, le pubblicazioni al di fuori del periodo campionate e che non sono state pubblicate in portoghese.

Al fine di ampliare le conoscenze sulla letteratura nazionale, la nostra ricerca bibliografica si è basata sulla ricerca di articoli nazionali, monografie, tesi, dissertazioni e libri che affrontano, in modo contestualizzato, il piano salariale. Questo filtro è stato elaborato dal titolo e dell'abstract di articoli scientifici, tesi, dissertazioni, monografie e libri.

I descrittori più efficienti erano "stipendio e piano di insegnamento", "piano salariale e formazione professionale e "piano salariale e insegnante". L'altro, "piano salariale ed educatore", "salario minimo e lavoratore dell'istruzione", "piano salariale e istruzione" ha restituito, insieme, 12 risultati. Alla fine, la ricerca ha totalizzato 60 produzioni bibliografiche da analizzare.

Tra le fonti di ricerca utilizzate, Google Scholar e Google Libri sono tornati con un volume di dati più elevato. Pertanto, è stato utilizzato il criterio di classificazione per pertinenza del contenuto seguito dalla selezione delle prime dieci opere che 
contemplano i criteri e gli obiettivi di questa ricerca. II periodo di tempo era dal $1^{\circ}$ gennaio 2008 al $1^{\circ}$ gennaio 2021, giustificato dalla data di inizio del pavimento e allungamento fino al periodo di modifiche nella legge FUNDEB.

Riconosciamo la limitazione dei criteri di ricerca, anche se comprendiamo che questo è uno sforzo per collegare la ricerca e le discussioni in linea con i progressi delle discussioni sul piano salariale nazionale. Articoli internazionali, notizie, produzioni che non si occupavano di salary floor nel contesto brasiliano o che lo affrontavano in modo marginale non sono stati considerati.

\section{LA PRODUZIONE BRASILIANA E LE SUE CARATTERISTICHE}

Sebbene gli approcci e il consolidamento delle politiche di apprezzamento degli insegnanti si siano evoluti per decenni, è dalla metà del 2010 che la discussione nell'area del piano salariale e della retribuzione degli insegnanti guadagna maggiore corpo, il che riflette la crescita delle pubblicazioni, sia in riviste scientifiche che in libri specializzati.

\section{A. Numero di ricerche nel corso degli anni}

Nel corso degli anni, le discussioni sulla legge PSPN sono state centralizzate nell'attuazione della politica. Analizzando le produzioni nell'arco di 13 anni, abbiamo osservato un aumento delle pubblicazioni negli anni 2016 e 2019, rispetto agli altri anni di pubblicazioni. C'è un aumento nel 2016, la cui possibile correlazione sono le elezioni comunali e i cambiamenti che potrebbero venire dalle nuove amministrazioni comunali. Un altro possibile rialzo nel 2019 sembra coincidere con il movimento sociale e l'articolazione sul Fondo Nazionale per lo Sviluppo dell'Istruzione FUNDEB, che aveva, fino a quel momento, carattere provvisorio.

RC: 101663

Disponibile in: https://www.nucleodoconhecimento.com.br/formazione-it/retribuzionedocenti 
Figura 1 - Numero di ricerche nel corso degli anni

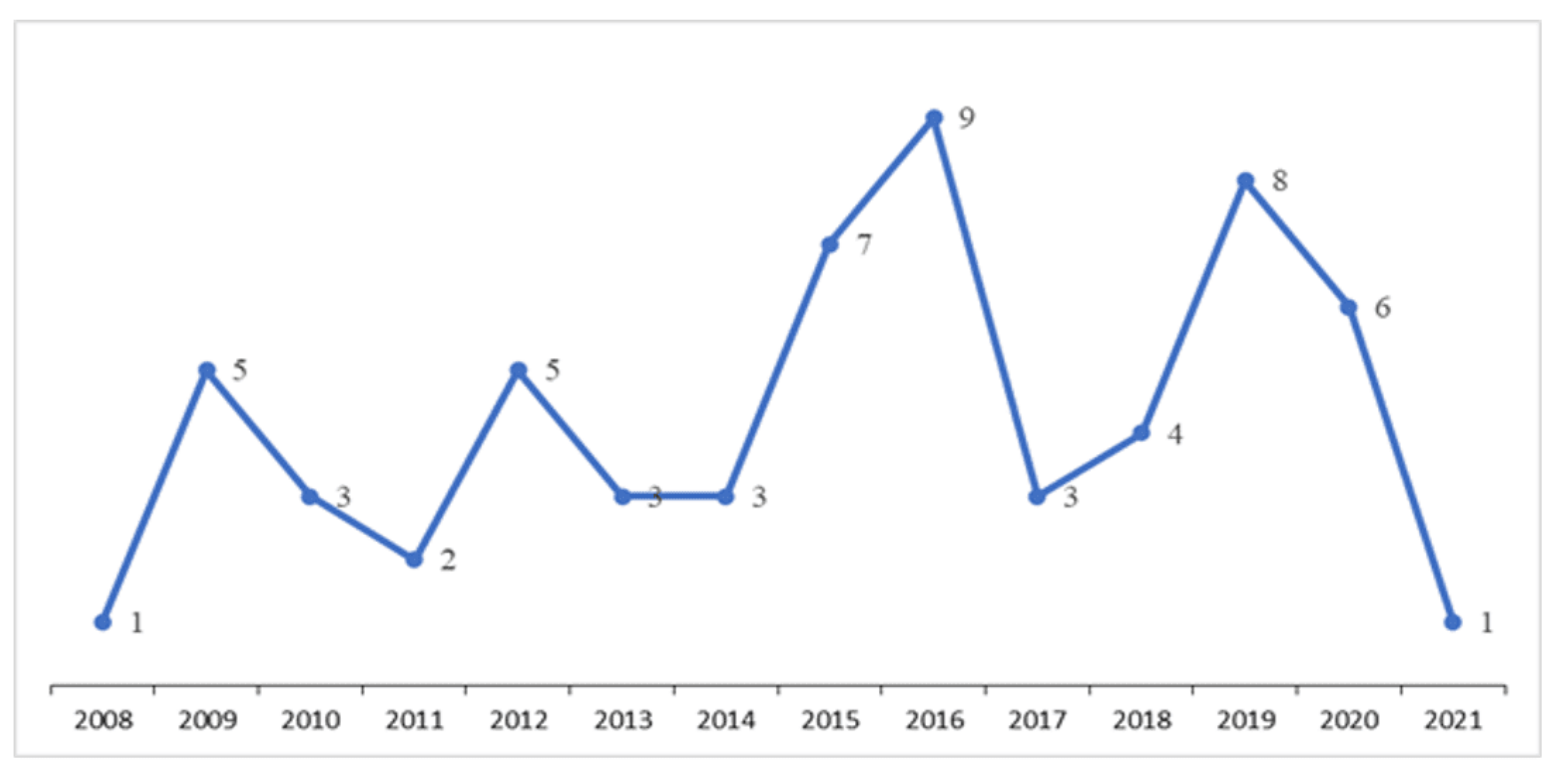

Fonte: Elaborazione propria (2021).

B. Tipi di pubblicazione

In generale, il tipo predominante di pubblicazione sono articoli e libri, che rappresentano l' $86,7 \%$ del totale di questa indagine. Questi studi sono stati per lo più guidati da produzioni nel settore dell'istruzione. Tesi, dissertazioni e monografie avevano una bassa rappresentatività nell'insieme totale di questa indagine in relazione ad articoli e libri.

RC: 101663

Disponibile in: https://www.nucleodoconhecimento.com.br/formazione-it/retribuzionedocenti 
Grafico 2 - Tipi di pubblicazione

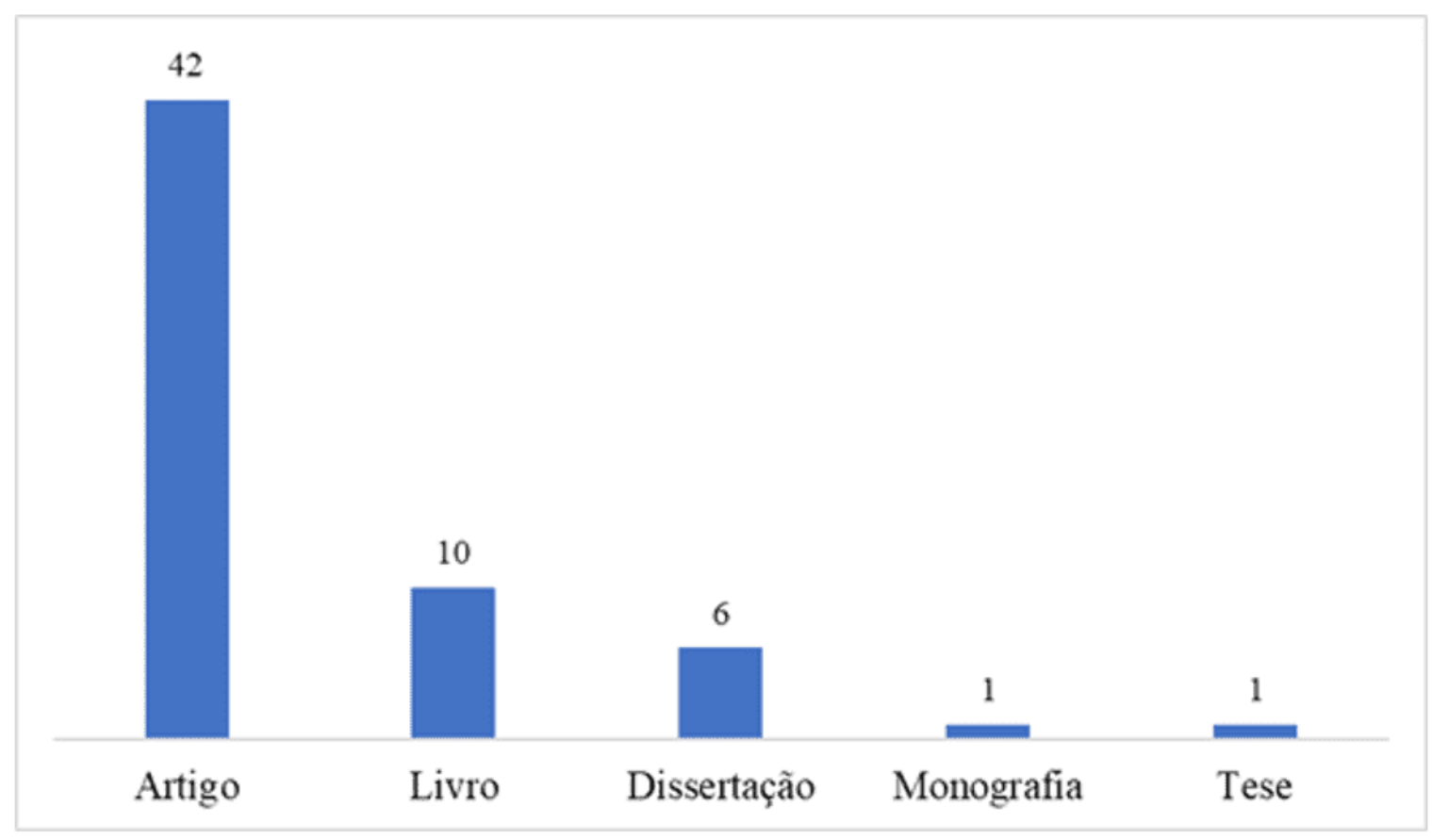

Fonte: Elaborazione propria (2021).

C. Aree di pubblicazione

La ricerca accademica in Brasile sul piano salariale è centrata nel settore dell'istruzione, raggiungendo il tasso dell' $80 \%$ (48 pubblicazioni). Del numero totale di pubblicazioni sul piano salariale nel settore dell'istruzione, 32 sono articoli e 10 sono libri. Pertanto, prevale la produzione di articoli e libri. Inoltre, le aree di Scienze Sociali e Scienze Politiche insieme rappresentano l'11,7\% (7 pubblicazioni). 
Grafico 3 - Aree di pubblicazione

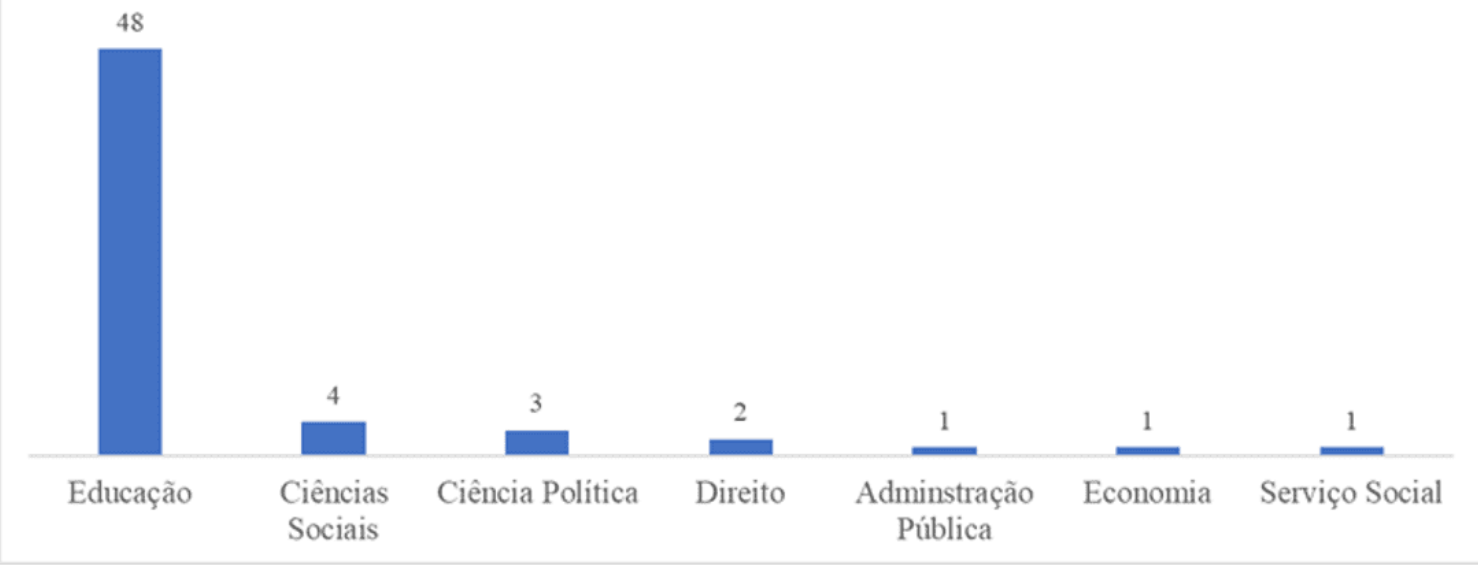

Fonte: Elaborazione propria (2021).

Figura 4 - Aree di pubblicazione per tipologia di pubblicazione

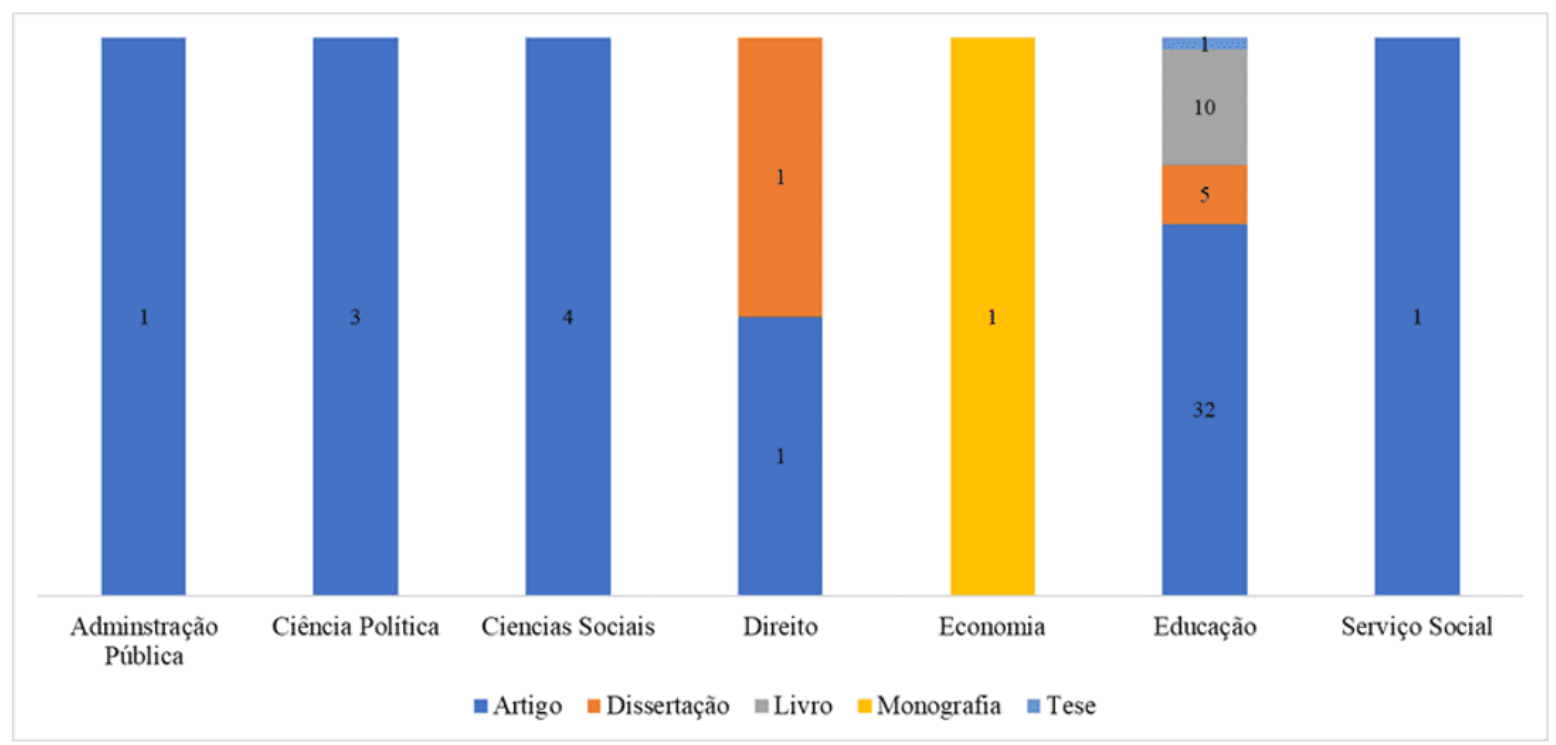

Fonte: Elaborazione propria (2021).

d. Principali aree di pubblicazione

Espandendo l'analisi della produzione accademica nazionale, abbiamo osservato se i luoghi di pubblicazione di articoli, libri, ecc. Erano regionali o nazionali.

RC: 101663

Disponibile in: https://www.nucleodoconhecimento.com.br/formazione-it/retribuzionedocenti 
Figura 5 - Regioni di pubblicazione

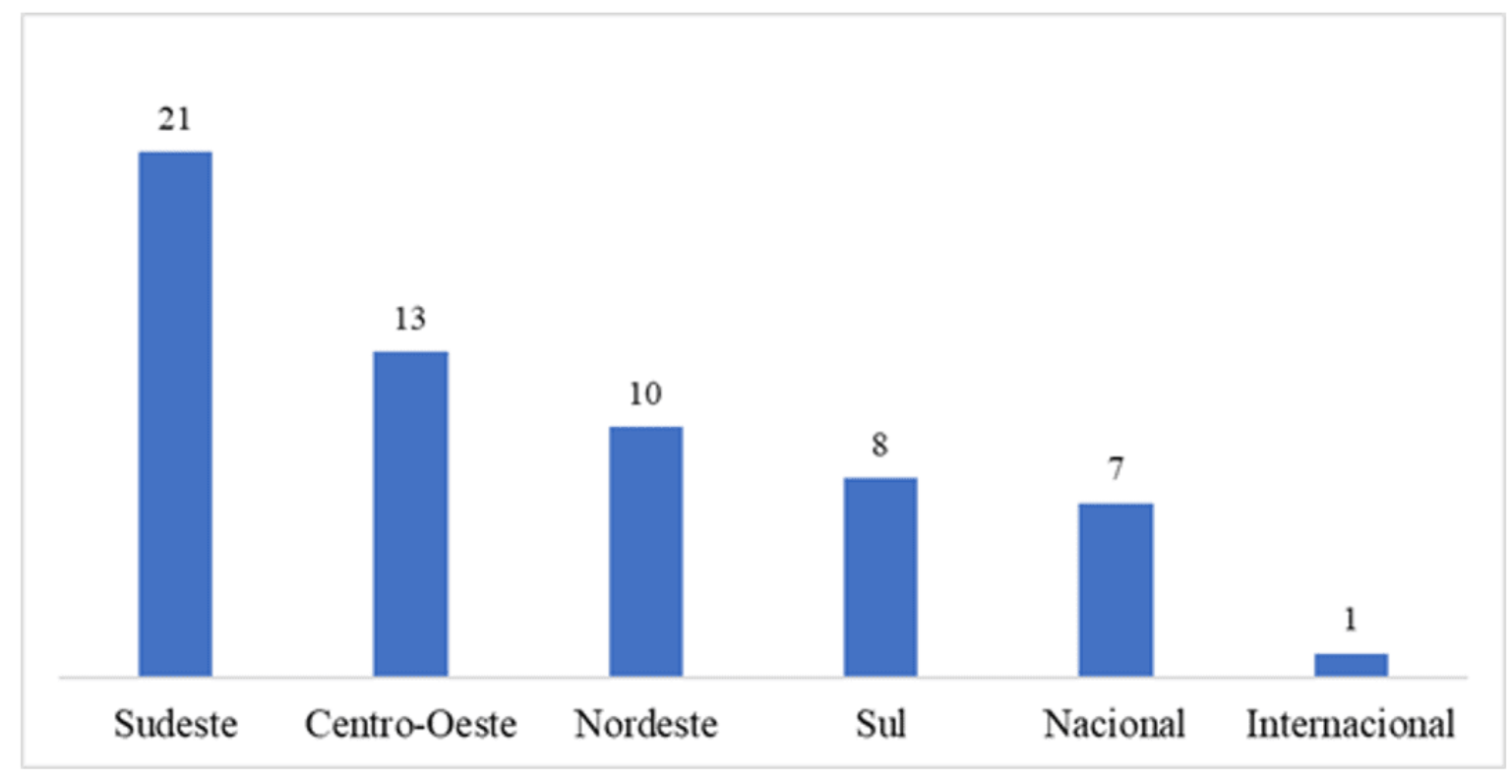

Fonte: Elaborazione propria (2021).

La ricerca bibliografica indica la regione sud-orientale del paese come uno dei principali luoghi di pubblicazione. Oltre al carattere nazionale, anche le regioni del Midwest, del Nord-Est e del Sud sono preponderanti, in ordine decrescente.

e. Principali luoghi di studio delle pubblicazioni 
Figura 6 - Regioni di studio delle pubblicazioni

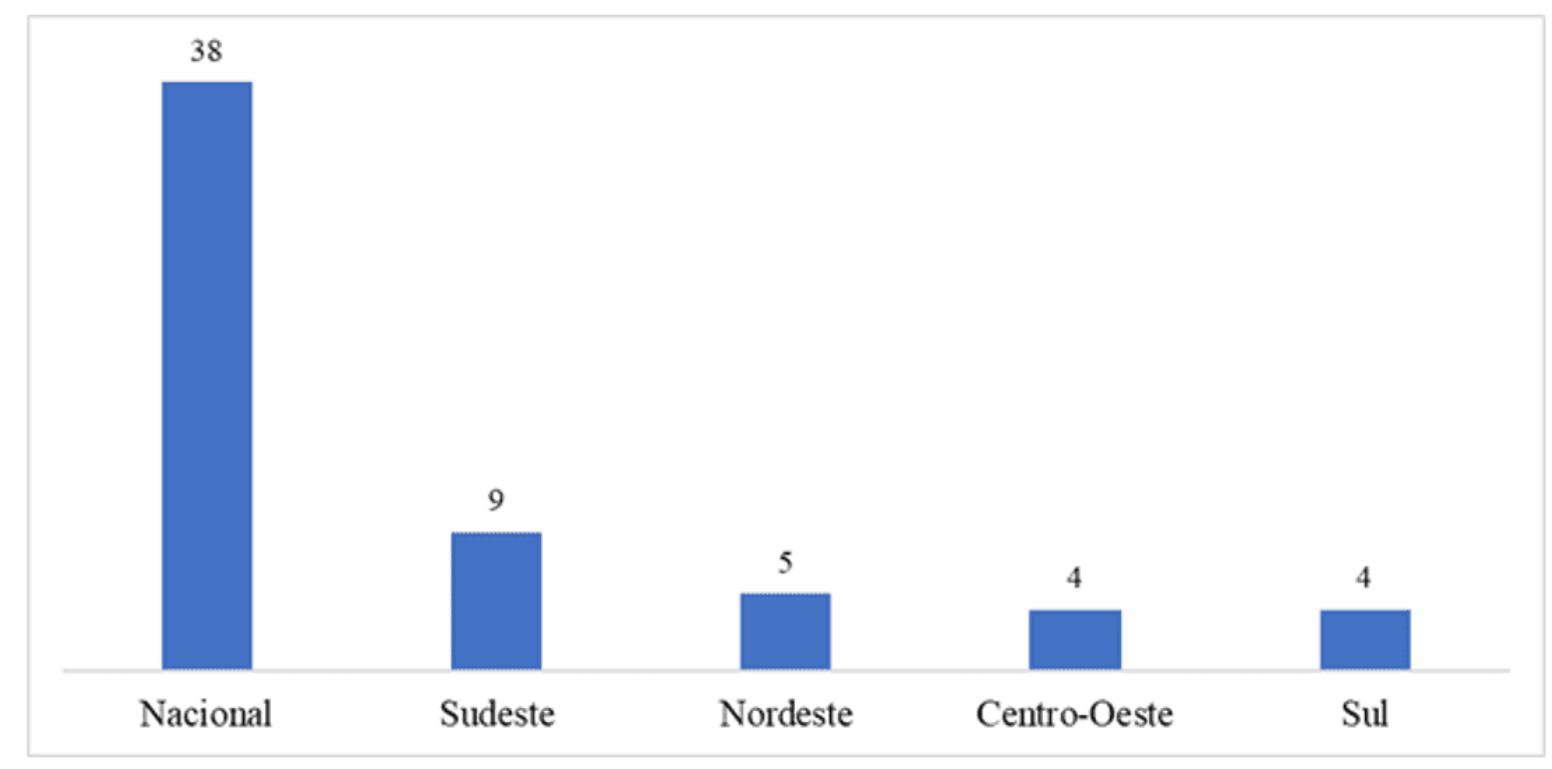

Fonte: Elaborazione propria (2021).

Nello specifico, analizzando i titoli e gli abstract delle pubblicazioni, abbiamo verificato I'andamento degli studi sulla legge PSPN a livello nazionale $(63,3 \%)$. Le analisi regionali comprendono circa il $36,7 \%$ di tutte le pubblicazioni. La regione sudorientale continua ad essere al centro di molti studi e, come abbiamo identificato, spiccano in particolare gli stati di San Paolo e Minas Gerais. Successivamente, emerge la regione nord-orientale, che rappresenta l' $8 \%$ del totale delle pubblicazioni, e vale la pena menzionare gli Stati di Bahia, Rio Grande do Norte e Piauí come fulcro dell'analisi degli studi regionali sul piano salariale. 
Grafico 7 - Studio di pubblicazione UF

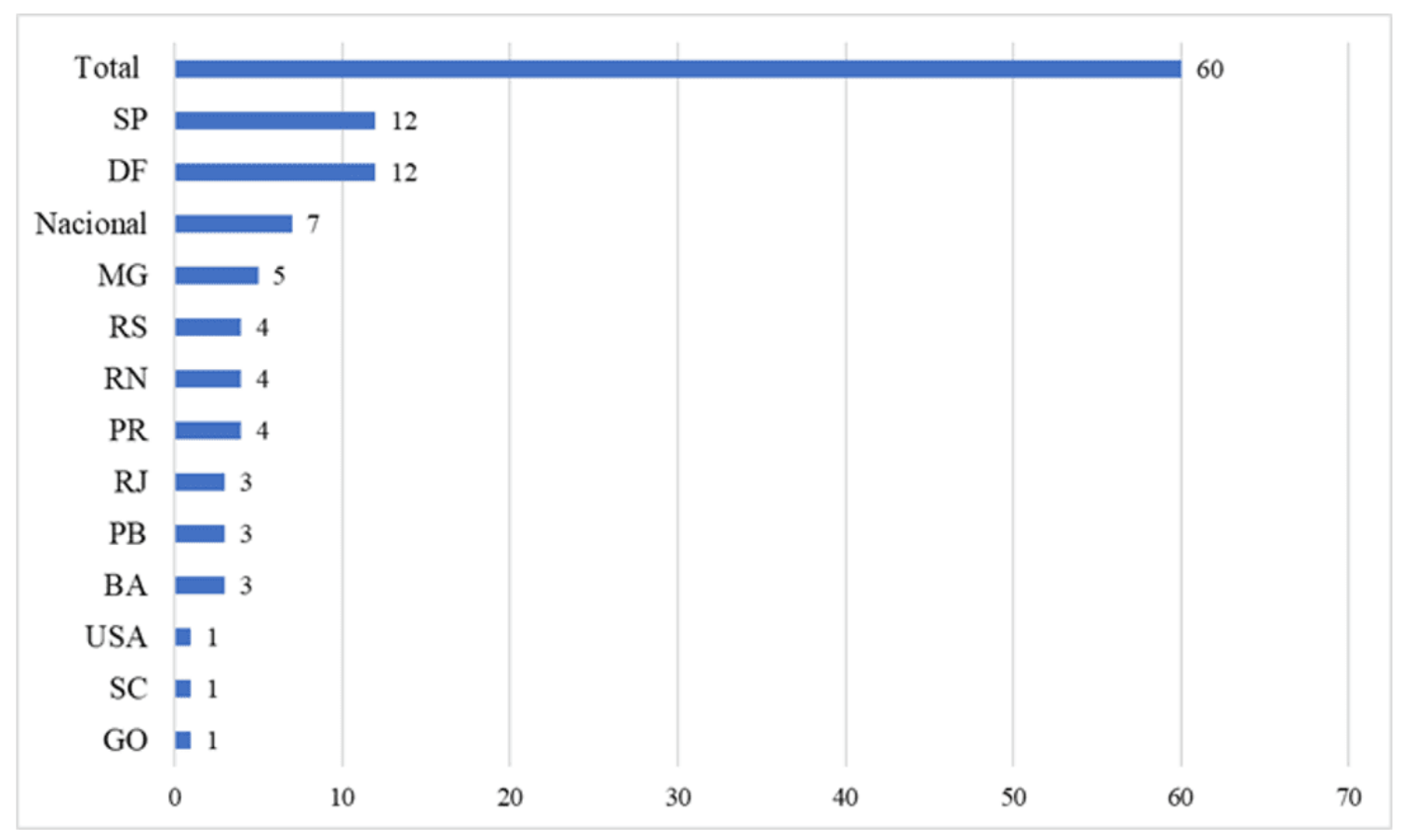

Fonte: Elaborazione propria (2021).

\section{f. Autori principali}

Dall'identificazione di articoli, monografie, dissertazioni, tesi e libri, è stato possibile sollevare i nomi degli autori che li hanno grafici. Vale la pena ricordare che quando l'opera aveva la co-paternità, come in alcuni libri specifici, venivano elencati i nomi di tutti.

Gli autori sono stati quindi elencati e raggruppati per ottenere il numero di volte che un determinato autore ha pubblicato. Per fare ciò, abbiamo creato un elenco con questi autori e sviluppato una nuvola di parole per visualizzare quelle più importanti. La nuvola di parole sottolinea, in modo direttamente proporzionale, la frequenza di apparizione delle parole. Quindi, possiamo osservare che i dieci autori con il maggior numero di pubblicazioni sono: Maria Dilnéia Espíndola Fernandes, Andréa Barbosa Gouveia, Márcia Aparecida Jacomini, Claudio Pinto Nunes, Eliara Cristina Nogueira da Silva Teixeira, Ana Paula Santiago do Nascimento, Andreza Barbosa, Áurea de 
Carvalho Costa e Dalila Andrade Oliveira. La classifica degli autori aiuta nella delimitazione degli autori e della bibliografia sul piano salariale. Possiamo vedere che la paternità è prevalentemente femminile.

Figura 1 - Principali autori

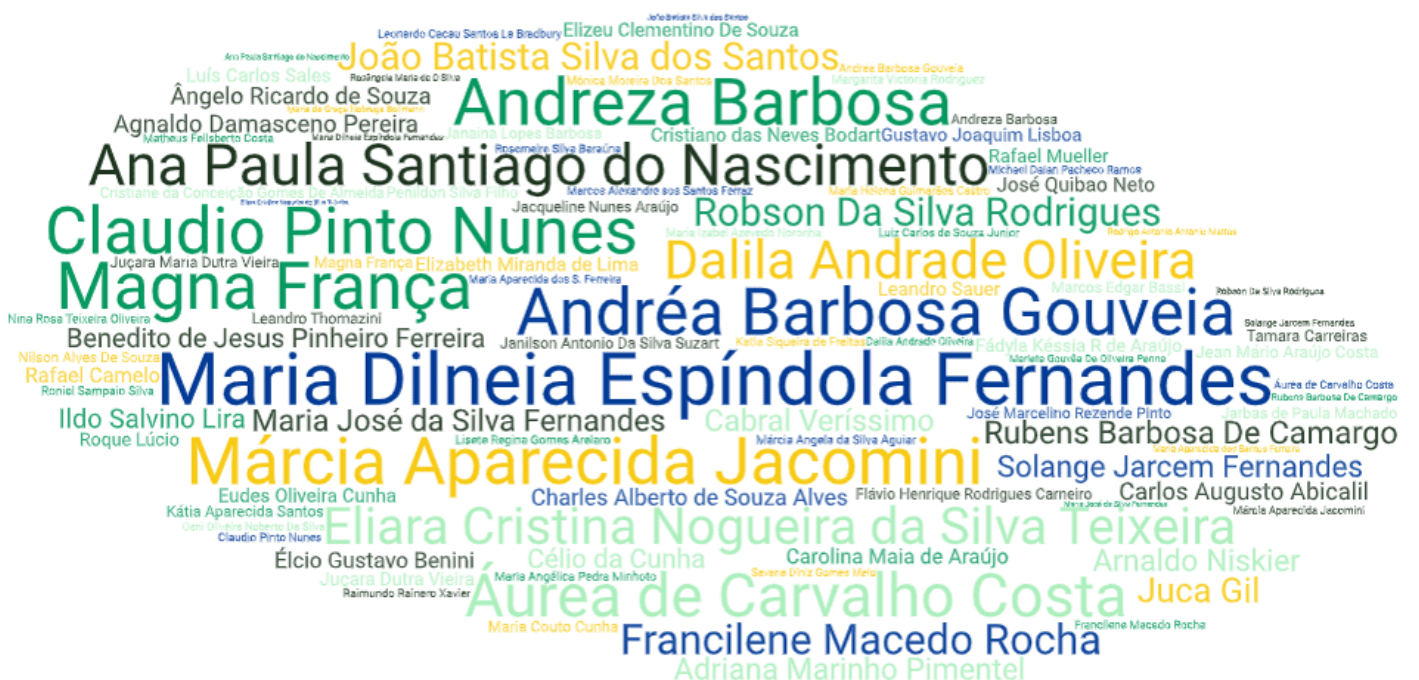

Fonte: Elaborazione propria (2021).

\section{IL PIANO SALARIALE PROFESSIONALE NAZIONALE E L'APPREZZAMENTO DEGLI INSEGNANTI: COSA DICONO GLI ACCADEMICI}

La revisione della letteratura ha carattere qualitativo ed esplorativo ed è stata prodotta per supportare gli studi di dottorato di questo ricercatore sull'attuazione della legge 11.738/2008, che ha creato il Piano salariale professionale nazionale - PSPN. Per contestualizzare la ricerca, è importante sapere come è stato affrontato il tema relativo alla retribuzione degli insegnanti nelle produzioni accademiche. Si tratta di una ricerca che inizialmente aveva l'unico scopo di contestualizzare, situando gli autori sulle posizioni produttive e accademiche, ma ha permesso di confrontare la produzione di discorsi in politica educativa e la verifica della loro applicazione pratica nel mondo accademico. Per questo lavoro, abbiamo selezionato i dieci articoli degli

RC: 101663

Disponibile in: https://www.nucleodoconhecimento.com.br/formazione-it/retribuzionedocenti 
autori che hanno prodotto di più sul pavimento, la retribuzione degli insegnanti e il rispetto della legge PSPN, tra il 2008 e il 2021. Questi articoli affrontano la remunerazione degli insegnanti e le loro relazioni con i fondi di finanziamento dell'istruzione di base.

Dopotutto, cosa dicono gli accademici sulla retribuzione degli insegnanti, considerando che ci sono stati quasi due secoli di lotte per il Piano salariale professionale nazionale?

I| 15 ottobre 1827, Dom Pedro I stabilì, nell'articolo 3 della legge sulle scuole di lettere, il primo piano salariale per l'insegnante, ma questo non fu mai applicato. Dalla Costituzione del 1988, le discussioni sindacali e accademiche hanno acquisito contorni sempre più pronunciati. Pochi esperti di educazione hanno scollegato la lotta per la democratizzazione della lotta per la scuola pubblica gratuita, con insegnanti con una solida formazione iniziale e una retribuzione decente. II 16 luglio 2008 , l'allora presidente Lula ha approvato la legge $11.738 / 2008$, che ha istituito il Piano nazionale degli stipendi professionali per i professionisti dell'istruzione pubblica di base, stabilendo un valore da adeguare obbligatoriamente ogni anno e "al di sotto del quale l'Unione, gli Stati, il Distretto federale e i Comuni non saranno in grado di fissare lo stipendio iniziale delle carriere dell'insegnamento pubblico dell'istruzione di base, per un viaggio di massimo 40 (quaranta) ore settimanali" (BRASIL, 2008). Sebbene il piano sia stato istituito per legge dal 2008, più di 10 anni dopo la sua sanzione in più della metà dei comuni, più della metà dei comuni brasiliani viola la legge floor, senza alcuna punizione.

Ci sono stati due secoli di lotte degli insegnanti per la creazione del piano salariale per il magistero, ma ci sono stati ancora tentativi da parte di alcuni governatori di impedire la loro sanzione, che fortunatamente sono stati frustrati.

Nel corso di un lavoro di consulenza svolto per l'UNESCO/MEC, abbiamo riscontrato che su 5.570 comuni, solo il $30 \%$ ha rispettato integralmente la legge 11.738/2008, cioè pagando il piano iniziale, creando nuovi piani di carriera, garantendo il $30 \%$ del 
carico di lavoro per studi e pianificazione, oltre a garantire una solida formazione iniziale e l'ingresso tramite gara pubblica. Pertanto, abbiamo deciso di studiare l'implementazione del piano salariale, e nel caso della tesi di dottorato[4] che stiamo sviluppando, in particolare sullo stato di Bahia, che è il $4^{\circ}$ stato più grande della Federazione e il più grande nel nord-est brasiliano. Inizialmente, per sostenere gli studi della tesi citata sullimplementazione del piano nei 417 comuni di Bahia, abbiamo cercato una base di ricerca bibliografica che ha raggiunto decine di studi. Per questo articolo, presentiamo i dieci autori che hanno prodotto di più sull'argomento in questo periodo. In questo contesto, lo scopo di questo articolo è quello di presentare lo sviluppo di questa linea di ricerca, che consideriamo rilevante. Evidenziamo le sue questioni centrali, i suoi progressi e limiti, in breve, lo stato dell'arte della letteratura su PSPN.

Nonostante il dilemma che coinvolge le influenze della politica sulle politiche pubbliche per gli insegnanti e per l'istruzione di qualità, in un paese di "democrazia ristretta", come ha sottolineato Florestan Fernandes (1987, p. 160), insistiamo sulla ricerca di domande che ci permettano di identificare il posto occupato dall'apprezzamento dell'insegnante e dall'educazione nell'universo accademico. Per rispondere alla domanda sull'interesse degli studi condotti sull'attuazione del Piano salariale professionale nazionale - PSPN e se questi indicano le ripercussioni del piano salariale per la valorizzazione del magistero, nel periodo 2008-2021, abbiamo cercato articoli, monografie, tesi e tesi nell'area delle scienze sociali o dell'area dell'istruzione.

Negli ultimi quarant'anni, autori più importanti, sebbene pochi, sono stati impegnati nell'analisi degli statuti, dei programmi e delle pratiche dei manager e hanno cercato di comprendere l'influenza dei partiti politici nello spiegare le variazioni nei risultati delle azioni governative nel settore dell'istruzione. In questo periodo, indubbiamente, gli studi sulle pratiche e le politiche educative arrivano includendo l'insegnante, prima quasi invisibile. Prova ne sono stati i capitoli della Costituzione federale e delle Costituzioni statali che evidenziano l'apprezzamento dell'insegnante; la legge delle linee guida e delle basi dell'educazione nazionale; la definizione delle Linee Guida RC: 101663

Disponibile in: https://www.nucleodoconhecimento.com.br/formazione-it/retribuzionedocenti 
Nazionali per la Formazione degli Insegnanti; i Piani Educativi Nazionali del Brasile PNE (2011-2020) e PNE (2014-2024) e la Legge 11.738/2008, che ha creato il piano salariale, documenti per i quali la qualità dell'istruzione e l'apprezzamento degli insegnanti costituiscono sfide reali. Nel nuovo Piano Nazionale dell'Educazione del Brasile - PNE (2014-2024), dei 20 obiettivi delineati, 4 riguardano l'insegnante, sebbene i Piani Educativi Nazionali siano stati messi da parte dai governanti in ambito federale, statale e municipale, e si stiano trasformando in meri protocolli di intenzioni.

Successivamente, passiamo alla ricerca degli accademici sul PSPN, sulla sua attuazione, remunerazione e apprezzamento del magistero.

L'Unesco ha pubblicato, nell'ultimo decennio, due opere mozzafiato: Professores do Brasil: impasses e desafios (2009), di Bernardete Angelina Gatti e Elba Siqueira de Sá Barretto, e Políticas Docentes - um estado da arte (2011), di Bernardete Angelina Gatti, Elba Siqueira de Sá Barretto e Marli Eliza Dalmazo Afonso de André. Questi studi portano una radiografia dei lavoratori dell'istruzione. Professores do Brasil: impasses e desafios (2009) mostra la dimensione della sfida che è la valorizzazione degli insegnanti e sottolinea che "gli insegnanti sono tra i gruppi professionali più numerosi". E ci sono ancora "le sfide del superamento delle convenienze politiche e dell'adozione di strategie articolate tra i diversi organismi di formattazione". In conclusione, gli autori usano la parola "trama", che è una sfida teorica ed è nell'ambito del nostro lavoro. Nel 1992 abbiamo pubblicato la monografia dal titolo "A trama da ignorância" (LIMA, 1996), in cui abbiamo analizzato i discorsi delle autorità brasiliane sull'insegnante, nell'Impero e nella Repubblica, confrontandoli con le efficaci politiche di valorizzazione dell'insegnante. Nel 2011, i ricercatori Bernardete Angelina Gatti, Elba Siqueira de Sá Barretto e Marli Eliza Dalmazo Afonso de André hanno pubblicato lo studio Políticas Docentes no Brasil uno stato dell'arte, pubblicato dall'UNESCO e di grande portata. In questo studio, gli autori mappano e analizzano le politiche didattiche applicate nelle diverse sfere federative - Unione, Stati e Comuni - e mettono al centro della discussione la formazione iniziale e continua dell'insegnante, la carriera di insegnamento e la RC: 101663

Disponibile in: https://www.nucleodoconhecimento.com.br/formazione-it/retribuzionedocenti 
valutazione degli insegnanti. Nelle conclusioni, si parla della diversità delle proposte per l'apprezzamento degli insegnanti, ma non si fa riferimento alla piena attuazione o meno della legge $11.738 / 2008$, che ha creato il Piano salariale professionale nazionale - PSPN -. E avvertono:

Se não houver aderência das propostas às políticas próprias dos estados e dos municípios, no caso das políticas federais, e destes últimos, no caso das políticas estaduais que a eles se estendem, ficam comprometidas a possibilidade de desenvolvimento profissional dos docentes e a sustentação das conquistas adquiridas (GATTI; BARRETO; ANDRÉ, 2011, p. 266).

II dibattito accademico sulla carriera e la retribuzione dell'insegnamento è stato oggetto di ricerche da parte degli autori Rubens Barbosa de Camargo, Andréa Barbosa Gouveia, Juca Gil e Maria Angélica Pedra Minhoto (2009). In questa ricerca, gli autori analizzano le relazioni tra i risultati del fundef sulla base di indicatori economici e la remunerazione degli insegnanti, avendo come parametri i riferimenti legali sul Piano salariale professionale nazionale. Per questo, gli autori hanno utilizzato "indicatori economici nazionali, stipendi degli insegnanti nelle reti statali e valori dell'anno studentesco Fundef". E hanno concluso che le variazioni salariali sono legate a ciascuno degli stati, presentando un'inflessione positiva verso la ricomposizione dei salari, al di sopra dell'inflazione. Evidenziano la "necessità di ulteriori studi per comprendere le dinamiche della remunerazione". Sulla necessità di ulteriori studi per sostenere la fissazione del piano salariale, vale la pena chiedersi se gli esperti hanno dimenticato che i padroni hanno aspettato due secoli per conquistare il piano salariale stabilito dalla legge, per l'intero paese.

Per gli autori, "la retribuzione degli insegnanti, oltre a dover essere meglio 'decifrata', per fornire un adeguato supporto alla concezione di un PSPN, perché determinata da fattori (esterni e interni) che coinvolgono interessi diversi" (CAMARGO et al., 2009). Tutto questo "per esporre la sua massima dimensione esplicativa, rivelandosi come espressione concreta di un rapporto di forze in disputa di progetti di società, di scuola, di uomo, di valorizzazione dell'insegnamento: insomma, come lotta politica" (CAMARGO et al., 2009, p. 360). Quali sono gli interessi di coloro che si muovono 
verso la mancata applicazione della Legge che ha istituito il piano salariale? Le dispute su cui Florestan Fernandes, tra gli scettici di destra o di sinistra che aiutano poco, quasi sempre sabotare l'istruzione in Brasile, sarebbero espresse qui?

In un articolo del 2010, Santiago do Nascimento, Amorim e Camargo (2010) hanno analizzato la remunerazione degli insegnanti nella rete statale di San Paolo all'inizio della loro carriera hanno evidenziato la loro composizione basata sul documento del Dipartimento di Stato dell'Istruzione intitolato Sistema informativo educativo Bollettino di monitoraggio del personale (novembre 2010). Con questo, hanno potuto verificare l'evoluzione degli adeguamenti dei pagamenti ricevuti dagli insegnanti, nel periodo tra il 1996 e il 2010.

Santiago do Nascimento, Amorim e Camargo (2010) hanno rilevato che "la remunerazione era composta da diversi bonus che rappresentavano oltre il $35 \%$ del totale". E che, in relazione al salario minimo, la retribuzione ha subito un appiattimento, da 3,6 nel 1996 a 2,4 nel 2010. Gli autori intendono contribuire alle discussioni sulla remunerazione degli insegnanti in Brasile analizzando i periodi da parte della direzione, dei governatori e / o dei segretari dell'istruzione. Nella gestione del governatore Mário Covas, la remunerazione dei professionisti PEB I è passata da $\mathrm{R} \$ 360,94$ nel 1996 a $\mathrm{R} \$ 710,00$ nel 2000 e nel 2001, in valori nominali. Confrontando questi valori, abbiamo notato che la remunerazione è cresciuta del $96,71 \%$ in cinque anni.

Sotto Alckmin, la remunerazione dei professionisti PEB I è passata da $R \$ 710,00$ nel 2001 a R \$ 1.144,39 nel 2005 (NASCIMENTO; AMORIM; CAMARGO, 2010). L'aumento è stato del $61,18 \%$ in quattro anni. La scadenza è passata da $R \$ 610,00 \mathrm{a}$ $R \$ 726,19$. Durante questo periodo, è stata pagata la più alta percentuale di bonus, con bonus che hanno raggiunto il $36,5 \%$ della retribuzione degli insegnanti.

Durante il periodo del governo di José Serra, i pagamenti iniziali degli insegnanti di PEB I sono passati da R \$ 1,144.39 nel 2008 a $R$ \$ 1,198.16 nel 2010, in valori nominali. Analizzando gli stipendi ( $R \$ 726,19$ e $R \$ 981,88$ ), abbiamo notato un 
aumento del 35,21\%. La rappresentatività dei bonus nel governo Serra è aumentata dal 36,5\% nel 2008 al 18,1\% nel 2010. Ciò significa che questo governo aveva una politica di incorporazione dei bonus nello stipendio, ma con una possibile perdita reale di potere d'acquisto, in base al rapporto con il salario minimo. La rappresentatività dei bonus nel governo Serra è aumentata dal 35,5\% nel 2008 al $17,4 \%$ nel 2010.

Pertanto, gli autori concludono che durante tutto il periodo studiato, se preso come indicatore del salario minimo (o del salario necessario di Dieese), è stata percepita una forte oscillazione. Tuttavia, se consideriamo gli anni 1996 e 2010, abbiamo notato che c'erano meno salari minimi, sia nello stipendio che nella retribuzione iniziale, indicando un appiattimento dei guadagni degli insegnanti di San Paolo.

Quando si tratta di politica di apprezzamento degli insegnanti, le tensioni sono alte. In un articolo pubblicato da Maria Dilnéia Espíndola Fernandes e Margarita Victoria, con il titolo "O processo de elaboração da Lei no 11.738/2008, Lei do Piso Salarial Profissional Nacional para carreira e remuneração docente: trajetória, disputas e tensões", gli autori salvano la recente traiettoria della costruzione della legge nazionale sul salario professionale (PSPN) per la carriera e la retribuzione degli insegnanti in Brasile, nel contesto delle riforme dello stato brasiliano. Hanno esaminato la legge federale, i documenti federali e sindacali e la letteratura sull'argomento. Hanno evidenziato uno dei più importanti scontri politici nella costruzione delle relazioni federative brasiliane in quel momento storico, che era un'azione diretta di incostituzionalità (Adin) contro la legge n. 11.738/2008, presentata dai governatori degli stati del Mato Grosso do Sul, Paraná, Santa Catarina, Rio Grande do Sul e Ceará. Nell'azione diretta di incostituzionalità, i governatori hanno messo in discussione il piano salariale nazionale degli insegnanti della scuola elementare pubblica, istituito dalla legge federale 11.738/2008, discutendo la portata dell'espressione "piano" (art. 2, caput e $\S 1^{\circ}$ ); la limitazione all'importo versato come stipendio base iniziale della carriera o l'estensione dello stipendio complessivo; e l'impostazione del carico di lavoro. E hanno asserito la violazione della legge di riserva di iniziativa del capo dell'esecutivo per disporre del RC: 101663

Disponibile in: https://www.nucleodoconhecimento.com.br/formazione-it/retribuzionedocenti 
regime giuridico del funzionario pubblico (art. $61, \S 1$, ii, c della costituzione); contrario al patto federativo (art. $60, \S 4$ e voce della costituzione); inosservanza della regola di proporzionalità: la giornata lavorativa e la retribuzione stabilita dalla legge. Gli autori hanno capito "che il pspnè impiantato in termini di concepimento". Con il Tribunale federale supremo, il più alto organo giuridico del paese, dopo aver approvato la legge n. 11.738/2008 (BRASIL, 2008a), la controversia legale è stata risolta, dando causa guadagno agli insegnanti, garantendo loro piani di carriera per l'insegnamento pubblico, stipendio base professionale e assegnazione del $30 \%$ del carico di lavoro per studi e pianificazione, oltre a entrare nella carriera esclusivamente mediante concorso pubblico di test e titoli. II 17 dicembre 2008, nel voto del ministro Joaquim Barbosa è stato scritto che "non è credibile presumere che gli Stati federati si oppongano alla riduzione delle disuguaglianze regionali, con il miglioramento delle condizioni in cui vengono erogati i servizi di istruzione pubblica". L'implementazione del piano nelle varie unità subnazionali ha dimostrato quanto sia difficile superare i localismi della tradizione culturale brasiliana nella costruzione storica del federalismo. Per loro, la fattibilità materiale del PSPN dipenderà anche da un grande sforzo da parte degli insegnanti di istruzione di base, nonché da possibili allineamenti nelle relazioni politiche attorno al modello di federazione in vigore. Lo scenario nazionale presenta situazioni molto diverse nell'affrontare la questione salariale dei professionisti dell'istruzione di base. Ciò che è in comune tra le unità federate sugli stipendi degli insegnanti è il basso stipendio dell'insegnante.

La specialista Dalila Andrade (2013) ha pubblicato lo studio dal titolo "As políticas de formação e a crise da profissionalização docente: por onde passa a valorização", in cui analizza la formazione dell'insegnante e il rapporto con la professionalizzazione. Nell'articolo, l'autore cerca di capire quali sono i fattori che determinano il miglioramento dell'istruzione. Critica "alcuni approcci che collocano l'insegnante come il principale responsabile del compito educativo e, di conseguenza, dei suoi risultati, spostando il focus delle strutture e delle relazioni sociali che coinvolgono il contesto scolastico" (ANDRADE, 2013, p. 51). 
Per Andrade (2013, p. 51), "a instituição da Lei do Piso Salarial Profissional Nacional e a criação do PARFOR são algumas das políticas orientadas na direção de maior valorização docente". In questa ricerca, l'autore "cerca di discutere alcune correlazioni tra formazione iniziale e formazione continua con remunerazione, nel tentativo di evidenziare le loro relazioni per un maggiore apprezzamento degli insegnanti" (ANDRADE, 2013, p. 51). E conclude che le attuali politiche educative mirano a soddisfare le richieste di un maggiore apprezzamento degli insegnanti. Dopo LDB 9394/96, il livello di istruzione degli insegnanti è aumentato in modo significativo. Tuttavia, la legislazione è stata modificata consentendo all'insegnante con un'istruzione di scuola superiore di diventare, in alcune entità federali, una regola e non un'eccezione. In questo l'autore osserva una battuta d'arresto nel piano legale. Conclude, con una certa apprensione, mostrando preoccupazione per un allontanamento dai pilastri delle politiche: carriera; condizioni di lavoro e retribuzione, con un possibile passaggio "a un modello di responsabilità degli insegnanti per la loro formazione, in particolare la continua, come forma di crescita individuale e professionale" (ANDRADE, 2013, p. 69). "C'è una sensazione generale che la professione di insegnante subisca un processo di svalutazione" (ANDRADE, 2013, p. 51). L'analisi dei dati della ricerca ha dimostrato che la correlazione tra istruzione iniziale superiore (titolazione) e retribuzione è sicura. A differenza della formazione continua, che non presenta una correlazione sicura. Questo propone di ripensare le politiche di formazione continua. L'autore sottolinea che affrontare la crisi della carriera didattica richiede misure efficaci per rafforzare la carriera, sia nelle forme di ingresso che nella permanenza dell'insegnante, nella carriera, che presuppone condizioni di lavoro e retribuzione.

Vediamo che le attuali politiche educative dovrebbero cercare un'articolazione tra politiche formative e condizioni di professionalizzazione, dando maggior peso a una solida formazione iniziale nella definizione e certificazione della professione. L'ex ministro dell'Istruzione Rossieli Soares, del governo del presidente Michel Temer, ha presentato, al Senato federale, il 15 maggio 2018, un documento intitolato "Panorama da Educação Brasileira". Nel documento, il MEC ha dichiarato 57 milioni

RC: 101663

Disponibile in: https://www.nucleodoconhecimento.com.br/formazione-it/retribuzionedocenti 
di iscrizioni alla rete pubblica, ma ha denunciato la bassissima qualità dell'istruzione pubblica, indicando tra le cause l'alta percentuale di insegnanti con una formazione inadeguata, che va dal $30 \%$ al $40 \%$. E la metà degli insegnanti di matematica non aveva alcuna formazione sul campo. L'ex ministro ha anche riferito che l'80\% degli insegnanti di istruzione di base sono formati in istituzioni private. Nei corsi di pedagogia, delle 690.780 iscrizioni, l' $80 \%$ di queste sono in rete privata. Secondo Rossieli, le università federali devono capire che la formazione degli insegnanti è una strategia di sviluppo nazionale.

La ricercatrice Maria Dilnéia Espíndola Fernandes (2013), nell'articolo "A valorização dos profissionais da educação básica no contexto das relações federativo brasileiro", analizza, da strumenti legali e legali, la valorizzazione dei professionisti dell'istruzione di base attraverso l'implementazione di Fundeb e del Piano salariale professionale nazionale (PSPN) nel contesto delle relazioni federative brasiliane. Dopo aver condotto una ricerca documentaria e aver esaminato la letteratura sull'argomento, l'autore ha scoperto che nelle relazioni federative brasiliane, le politiche educative potrebbero contribuire alla collaborazione federativa per quanto riguarda la riduzione delle disuguaglianze sociali. L'autore ha concluso che "l'esercizio del potere locale è ancora spesso un ostacolo al coordinamento federativo per quanto riguarda la politica educativa" e questo "determinerebbe il forte potere di indurre I'Unione di fronte, tra le altre situazioni, all'attuazione del PSPN" (FERNANDES, 2013).

Nel 2016, Andreza Barbosa e Maria José da Silva Fernandes, nell'articolo intitolato "Piso Salarial em São Paulo: desvalorização dos professores", pubblicato sulla rivista Retratos da Escola, hanno discusso l'attuale situazione degli stipendi e dell'orario di lavoro degli insegnanti nella rete pubblica statale nello Stato di San Paolo per verificare l'adeguatezza alla legge n. $11.738,2008$, che ha creato il piano salariale del magistero. Gli autori hanno identificato diverse prove che rivelano "che il governo statale aggira sistematicamente la legislazione federale, secondo l'orario di lavoro degli insegnanti alla legge sul pavimento, e paga, quell'anno, una retribuzione inferiore a quella legalmente stabilita, il che aumenta la svalutazione degli insegnanti" (BARBOSA; FERNANDES, 2016, pag. 243).

RC: 101663

Disponibile in: https://www.nucleodoconhecimento.com.br/formazione-it/retribuzionedocenti 
Il documento di ricerca intitolato "Financiamento da Educação e Luta Sindical: conflitos em uma grande rede de ensino", di Andréa Barbosa Gouveia e Marcos Alexandre dos Santos Ferraz, incentrato sull'impatto della legge nazionale sul salario professionale sulle controversie degli insegnanti a Curitiba con il manager della rete scolastica locale, ha analizzato le agende delle rivendicazioni del Sindacato degli insegnanti tra il 2008 e il 2012. Gli autori hanno focalizzato la loro attenzione su elementi relativi al pavimento e alla remunerazione e all'implementazione dell'attività di $1 / 3$ ora. È stato possibile osservare diversi modi di vocalizzare le richieste del pavimento e diversi impatti concreti sulla remunerazione. In certi momenti, causando l'appiattimento della carriera e, in altri, l'appiattimento del pavimento stesso. Per quanto riguarda l'attività oraria, questa cessa di essere una pretesa di organizzazione del lavoro e assume la caratteristica di un diritto equo. Gli autori hanno concluso che il modo in cui sono state costruite le agende dei reclami è cambiato dopo il riaggiustamento del pavimento - e che, dal 2012, "dopo aver cambiato il modo di spiegare il reclamo del pavimento, l'indice di aggiustamento inizia a concentrarsi sull'intera tabella degli stipendi, senza distinzione. Così, l'effetto appiattimento della carriera è contenuto" - e che l'implementazione di un'attività di $1 / 3$ ora diventa un tema importante, sorprendente nelle affermazioni. Negli ordini del giorno precedenti al 2011, il tempo di attività era limitato ad un'agenda di controllo del rispetto della legislazione comunale già in vigore. Fino al 2011, le richieste di adeguamento salariale seguivano la logica di sommare un insieme di indici percentuali: indice di inflazione del periodo, indice di guadagno reale, indice di perdita storica. E fino al 2011, l'adeguamento del livello 1 della carriera (cioè il pavimento) si comportava sempre in modo diverso dagli altri livelli. Cioè, il pavimento per l'insegnante laureato è superiore a quello che si verifica negli altri punti della tabella salariale (eccezione fatta all'anno 2010). Ciò significa che c'è stato apprezzamento del pavimento, ma con l'appiattimento della carriera. L'aspetto interessante è che nella somma degli anni 2009, 2010 e 2011, il livello 1 della carriera ha un adeguamento superiore rispetto all'adeguamento del piano nazionale nello stesso periodo. A partire dal 2012, questo concetto di somma di più indici è sostituito da un singolo valore nominale del pavimento. L'effetto di queste due 
distinte strategie è diverso quando si osserva l'esito finale dei negoziati. Gli autori concludono anche che l'agenda acquista la dimensione di rivendicare un diritto prescritto dalla legge e concludono affermando: "il caso specifico della Rete Municipale di Curitiba e SISMMAC mostra che gli insegnanti sono in continua valutazione dei suoi effetti, oltre a rivalutare le proprie tattiche e strategie di lotte per poter godere di un diritto che la società brasiliana ha concesso loro come equo" (GOUVEIA; FERRAZ, 2016).

Uno degli articoli più intriganti è stato pubblicato nel 2016 sulla rivista Pro-posições, con il titolo "Análise da carreira docente e valorização do magistério: condições de trabalho e desenvolvimento profissional", scritto da due accademici che sono nella lista dei dieci autori che hanno pubblicato di più sulla valorizzazione del magistero, Márcia Aparecida Jacomini e Marieta Gouvêa de Oliveira Penna. Gli autori hanno affrontato diversi aspetti relativi alle condizioni di lavoro degli insegnanti di istruzione di base in Brasile e al loro sviluppo professionale, utilizzando i dati della ricerca "Remuneração de professores de escolas públicas de educação básica: configurações, impasses, impactos e perspectivas", condotta in 12 stati brasiliani e nelle loro capitali. Dopo aver analizzato i piani di carriera, verificato la formazione richiesta per l'ammissione, l'orario di lavoro, gli incentivi per la formazione continua e l'avanzamento di carriera, gli autori affermano che "nonostante l'importanza degli insegnanti per la promozione di un'istruzione di qualità, diversi problemi devono ancora essere affrontati al fine di garantire condizioni di lavoro efficaci per gli insegnanti" (JACOMINI; PENNA, 2016). Gli autori concludono l'articolo citando una ricerca sullo status dell'insegnamento in paesi come Argentina, Brasile, Perù e Uruguay, di Emílio Fanfani (2007, apud JACOMINI; PENNA, 2016), in cui sottolinea che

toda política docente deve ser integral. Isto quer dizer que deve contemplar intervenções articuladas em pelo menos três dimensões: o recrutamento e a formação inicial e permanente, as condições de trabalho (divisão do trabalho pedagógico, carreira, contexto institucional, de trabalho, etc. e o sistema de estímulo e recompensa s materiais e simbólicas (salário e reconhecimento social).

RC: 101663

Disponibile in: https://www.nucleodoconhecimento.com.br/formazione-it/retribuzionedocenti 
In un altro paragrafo delle considerazioni finali, i suddetti autori concludono che "la ricerca presentata in Brasile sulle condizioni di lavoro dell'insegnante indica una svalutazione politica e sociale dell'insegnamento strutturando tale pratica professionale". E affermano, senza entrare nel merito, che "l'insegnante deve avere lo stipendio in linea con il valore e l'importanza che questi professionisti hanno nella società contemporanea". La ricerca indica la svalutazione degli insegnanti con la società contemporanea e che per cambiare questa situazione, è necessario riconoscere e valorizzare la società.

L'analisi delle "Intencionalidades das políticas de valorização docente que foram efetivadas nos governos FHC e LULA, a partir das políticas de Fundos (Fundef e Fundeb)" è stata oggetto di uno studio pubblicato da Teixeira e Nunes (2016). In questo articolo, gli autori esaminano la questione dello stipendio e della carriera degli insegnanti di istruzione di base attraverso l'analisi del posto occupato dal Piano salariale professionale nazionale (PSPN) nelle agende dei governi FHC e Lula. Hanno anche verificato se le politiche applicate da questi governi hanno contribuito al miglioramento dei salari degli insegnanti brasiliani. E concludono che il salario nazionale come misura di valorizzazione del magistero ha ricevuto approcci diversi nei governi FHC e Lula. II Fundef, nel governo FHC, sebbene abbia causato un miglioramento degli stipendi degli educatori, si è risentito "di un riferimento nazionale per l'inizio di una carriera, che ha indotto questo Fondo a non raggiungere il suo obiettivo di valorizzare l'insegnamento dell'istruzione di base". D'altra parte, Fundeb, nel governo di Lula, oltre a mantenere un minimo del $60 \%$ delle risorse per il pagamento degli insegnanti, ha istituito il periodo di un anno per la creazione del Piano salariale in una legge specifica, che è culminata nella legge n. 11.738/2008, che ha stabilito l'attuazione del Piano di posizione (TEIXEIRA; NUNES, 2016, p. 252).

In un articolo di Robson da Silva Rodrigues e Áurea de Carvalho Costa (2019) intitolato "Da Constituição Federal de 1988 ao Plano Nacional de Educação 20142024: ardilosas apropriações da noção de valorização do trabalho docente", gli autori concludono che le politiche attuate tra il 1988 e il 2014 hanno promosso la RC: 101663

Disponibile in: https://www.nucleodoconhecimento.com.br/formazione-it/retribuzionedocenti 
svalutazione del magistero. La ricerca aveva come riferimento teorico il materialismo storico-dialettico. Gli autori affermano che osservando l'attività educativa nelle pratiche quotidiane, "coloro che attuano la pratica educativa contraddicono le politiche educative che pretendono di mirare a valorizzare gli insegnanti. Questa contraddizione deriva dall'applicazione di nozioni di valorizzazione svuotate di significato assiologico". In questi casi, oltre alla legislazione statale, "il riconoscimento del valore dell'insegnante è legato a prescrizioni esterne sul proprio lavoro e che si traducono in premi sotto forma di retribuzione, benefici e progressione di carriera, legati alla cultura della performance" (RODRIGUES; CARVALHO, 2019, p. 231).

In uno studio condotto da Andréa Barbosa Gouveia e Maria Dilneia Espíndola Fernandes (2019), basato sui dati riportati dallAnnual Social Information List (RAIS, 2016) sugli stipendi degli insegnanti negli stati del Mato Grosso do Sul e del Paraná, è stato dimostrato che l'attuazione e il mantenimento del Piano salariale professionale nazionale erano dovuti all'introduzione del governo federale e alla conseguente azione sindacale. L'agenda del sindacato degli insegnanti incorporava la difesa del principio della valorizzazione professionale e costruiva la necessità di un Piano salariale professionale nazionale (PSPN) come elemento strutturante. La regolamentazione nazionale del PSPN è avvenuta nel 2008; tuttavia, a causa del patto federativo brasiliano, il rispetto della legislazione dipende dai governi locali. Ciò ha comportato un'ampia mobilitazione dei sindacati degli insegnanti in modo che la definizione di retribuzione possa incorporare la regola nazionale. Per gli autori, "lo scenario di crisi istituzionale installato nel 2015, seguito da un'agenda economica restrittiva per i diritti sociali, ha messo a rischio l'insieme delle condizioni duramente conquistate per la valorizzazione degli insegnanti" (GOUVEIA; FERNANDES, 2019, p. 89).

In un'indagine condotta presso il Belo Horizonte Municipal Education Network RMEBH, gli specialisti Francilene Macedo Rocha e Savana Diniz Gomes hanno concluso che "la legge PSPN, a differenza dell'apprezzamento dell'insegnamento da essa sostenuto, ha indotto una riduzione della retribuzione e una restrizione RC: 101663

Disponibile in: https://www.nucleodoconhecimento.com.br/formazione-it/retribuzionedocenti 
dell'orizzonte di lotte degli insegnanti per la carriera unica degli insegnanti RMEBH, conquistata nel 1996" (ROCHA; MELO, 2019, p. 3). Per gli autori, con la politica Piano salariale professionale nazionale, c'è stato un processo di precarietà del lavoro di insegnamento presso RMEBH, dalla segmentazione della carriera di insegnante in insegnante di scuola elementare e insegnante di educazione della prima infanzia, che ha rotto la logica di una carriera unica di i professori RMEBH. Questo articolo presenta una discussione sui cambiamenti apportati alla carriera e alla retribuzione degli insegnanti nella rete educativa comunale di Belo Horizonte (RMEBH) dal 2003, con la creazione della posizione di educatore della prima infanzia (EI), e dal 2008 in poi, con l'istituzione della legge nazionale sul piano salariale professionale (PSPN). II periodo studiato è stato dal 2003 al 2016. Per l'analisi, i ricercatori hanno utilizzato autori critici classici, come Karl Marx e Friedrich Engels, e contemporanei, come Oder dos Santos, João Bernardo e Ricardo Antunes, tra gli altri, come riferimento teorico. Si basavano sulla bibliografia sul territorio, sulla ricerca documentaria incentrata sulle leggi nazionali e comunali in materia di carriera e retribuzione e sulle pubblicazioni dell'Unione dei lavoratori dell'istruzione della rete pubblica municipale di Belo Horizonte (Sind-REDE/BH).

L'articolo "Remuneração docente: efeitos do Plano de Cargos, Carreira e Remuneração em contexto municipal", di Maria Dilnéia Espíndola Fernandes, Solange Jarcem Fernandes e Viviane Gregório de Campos (2016), pubblicato sulla rivista Ensaio: Avaliação e Políticas Públicas, è stato preparato da un caso di studio. In questo studio, gli autori hanno cercato di verificare l'effetto prodotto dai Piani di Lavoro, Carriera e Retribuzione (PCCR) attuati nel comune di Campo Grande, nel periodo dal 1996 al 2016, in termini di retribuzione salariale per un insegnante oltre i 20 anni di carriera. L'analisi dell'evoluzione della retribuzione dell'insegnante è stata fatta dall'esame dei loro buchi, rispetto al PCCR e al salario minimo. Gli autori hanno scoperto che c'era un impatto positivo sulla remunerazione della professione di insegnante, con il PCCR comunale obbligatorio, e che il grado e l'orario di lavoro erano importanti per la valorizzazione dell'insegnante. Tuttavia, il pagamento 
completo del PSPN (Piano salariale professionale nazionale) è rimasto, tuttavia, in una storia comune, che dovrebbe essere garantita anche attraverso il PCCR.

\section{ALCUNE CONSIDERAZIONI}

La raccolta di articoli sulla rilevanza degli studi riguardanti la piena attuazione del Piano salariale professionale nazionale - PSPN per i professionisti dellistruzione di base corrobora, in una certa misura, i risultati. Per quanto riguarda la svalutazione dell'insegnante e la comprensione del significato di valutare le ripercussioni del PSPN sulla sua valorizzazione, le conclusioni confermano gli studi che considerano i modelli istituzionali, le richieste sociali, le forme di governo, le politiche di partito e gli spettri politico-ideologici come influenzatori dei risultati, sebbene queste non siano le uniche cause. Più importante delle caratteristiche formali delle istituzioni statali e sociali è il modo in cui una configurazione istituzionale influenza le relazioni politiche.

La democratizzazione del paese, la costruzione di un insieme di leggi e l'osservazione del funzionamento delle istituzioni hanno trasformato lo studio delle politiche pubbliche. Ciò è evidente quando identifichiamo le variabili istituzionali formali o informali nell'analisi dei fattori determinanti delle politiche educative, come il comportamento della magistratura; l'osservazione della struttura federativa, con i suoi ostacoli; localismi in contrapposizione alla politica di collaborazione federativa; e gruppi di interesse e scontri di posizioni politico-ideologiche che sabotano le politiche educative. Allo stesso modo, la questione istituzionale serve a identificare le somiglianze tra le unità di analisi, consentendo di concentrarsi solo sulla variazione di altre caratteristiche desiderate, come i partiti, le elezioni e le condizioni socioeconomiche delle entità federative.

Abbiamo osservato un numero significativo di stati e comuni che applicano PSPN in base ai loro interessi e richieste sociali, spesso in mancanza della legge. In sintesi, la complessità delle politiche di partito ci ha imposto la necessità di creare una varietà di modelli e teorie per analizzare i loro processi e risultati, come è stato chiaro nelle discussioni di ogni articolo. Tali strategie analitiche, in diverse misure, si completano 
a vicenda e, allo stesso tempo, corroborano lo sviluppo congiunto e globale di questo campo di ricerca sulle politiche pubbliche per l'istruzione, in particolare sulla correlazione tra istruzione di qualità e apprezzamento degli insegnanti.

\section{G. Principali argomenti trattati}

La ricerca bibliografica ha incluso l'estrazione di abstract dei testi e soprattutto le parole chiave degli articoli, monografie, dissertazioni e tesi. Quando i libri sono stati analizzati, sono stati rimossi i principali argomenti di indicizzazione bibliografica. Agglutinante tutti gli argomenti trattati, generiamo la frequenza di ogni parola chiave.

La seguente nuvola di parole ti consente di classificare i temi su sei argomenti più rilevanti. Sono: politica educativa, lavoro di insegnamento, piano salariale, piano salariale professionale nazionale, valorizzazione dell'insegnamento e remunerazione degli insegnanti, che suggeriscono una grande correlazione con l'oggetto di studio di questo lavoro.

Figura 2 - Parole chiave

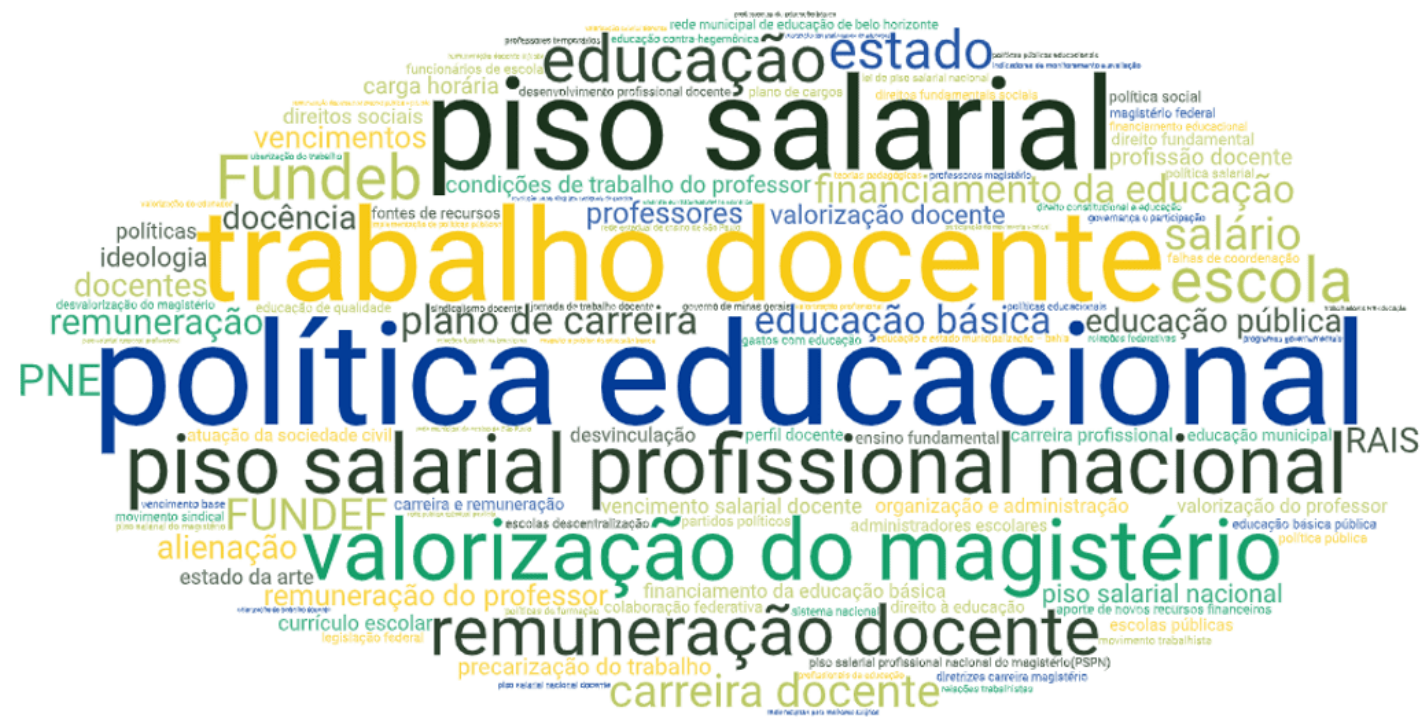

Fonte: Elaborazione propria (2021).

RC: 101663

Disponibile in: https://www.nucleodoconhecimento.com.br/formazione-it/retribuzionedocenti 
In cui pesano tante parole del cloud che esaltano la politica educativa, il lavoro di insegnamento, il piano salariale, il piano salariale professionale nazionale, l'apprezzamento dell'insegnamento e la remunerazione degli insegnanti, queste parole non sembrano mantenere la correlazione attesa nella produzione accademica. Questo perché non produce maggiori impatti sulla produzione di un numero significativo di tesi di master e tesi di dottorato, e questi studi sono concentrati in articoli o in indicizzazione bibliografica di libri.

Nell'esperienza di oltre quarant'anni di militanza nella classe dell'istruzione primaria, media e superiore - come leader sindacale nello stato di Bahia, vicepresidente della Confederazione nazionale dei lavoratori dell'istruzione (CNTE), deputato statale dal 1991 al 1999, consulente legislativo alla Camera dei rappresentanti (1999-2003), consigliere speciale del ministro dell'Istruzione (2003-2004), Consigliere speciale della Presidenza dell'INEP e Assistente Segretario della Formazione Professionale di MEC - questo ricercatore ha empiricamente incontrato la tibieza di molti esperti di istruzione e autorità educative, quando si tratta di difendere salari decenti per l'insegnante. Conosceva anche gli impatti di questa concezione così radicata nella tradizione culturale del Brasile nella produzione accademica poco significativa sul pagamento dello stipendio, come se questo fosse un tema minore.

In questi quattro decenni di militanza nell'educazione, questo autore ha incontrato con sgomento due rinomati intellettuali brasiliani che sostenevano una remunerazione decente per l'insegnante: il sociologo Florestan Fernandes e il filosofo Luiz Felipe Pondé. Florestan (1989, p. 58) considerava "la dereduzione salariale dell'insegnante di primo grado la più deleteria di tutte". E Florestan ha concluso: "pochi paesi riducono l'insegnante di primo grado a una condizione così vicina alla miseria relativa come il Brasile". Per lui, "lo stesso vale per il maestro di scuola superiore, esposto a una condizione di insicurezza e bassa retribuzione senza eguali. Questi gradi di istruzione sono pilastri dell'istruzione scolastica". E Felipe Pondé intervistato dal giornalista Thais Oyama, nel Programma Linhas Cruzadas della Fondazione Padre Anchieta (21/10/2021) ha denunciato che "trattare la professione dell'insegnante e l'educazione come un tema idealizzato, come un RC: 101663

Disponibile in: https://www.nucleodoconhecimento.com.br/formazione-it/retribuzionedocenti 
sacerdozio, è una strategia per mantenere l'insegnante povero e felice". II filosofo sottolinea che "è necessario dire questo, perché in Brasile la condizione lavorativa dell'insegnante pone fine alla vita di chiunque. Gli stipendi degli insegnanti sono tra i peggiori al mondo" (PONDÉ, 2021).

Pondé sottolinea che "questo trattamento produce persone scoraggiate; persone che soffrono molto per lavorare; gente che ha poco orizzonte" (PONDÉ, 2021). Pertanto, oltre ad essere nella nuvola di parole classificate, questo dovrebbe essere un tema centrale nelle tesi di laurea magistrale e nelle tesi di dottorato, non solo nell'area dell'istruzione, ma soprattutto nell'area del diritto pubblico. Dopotutto, non rispettare la legge sul salario è un'indicibile trasgressione della legge; dovrebbe essere oggetto di master e dottorati in Scienze Sociali e Politiche, poiché questo mette in discussione la Politica Nazionale dell'Educazione, la Costituzione Federale e la Legge sul Piano Nazionale dell'Educazione, sanciti nelle votazioni del Congresso Nazionale. Si precisa che il mancato rispetto della Floor Law rappresenta uno smantellamento della Politica Educativa, poiché 4 (quattro) dei 20 obiettivi dell'attuale PNE riguardano la valorizzazione della didattica. Inoltre, questo mette in discussione i programmi di partito, facendo un vero e proprio disservizio alla democrazia.

Le parole magiche usate per inibire discussioni, rivendicazioni e studi accademici sullo stipendio dell'insegnante sono: sacerdozio e corporativismo. Queste strategie hanno funzionato in modo secolare.

Pertanto, questo risultato di parole così espressive, nel nuvola - in cui spiccano piano retributivo, lavoro didattico, politica educativa, piano retributivo professionale nazionale, apprezzamento dell'insegnamento, retribuzione - non si articola con i risultati delle ricerche sui numeri e tipologie di pubblicazione estratte da abstract di testi, parole chiave di articoli, monografie, dissertazioni e tesi e libri, da cui sono stati tratti i principali temi di indicizzazione bibliografica. 


\section{CONSIDERAZIONI FINALI}

Quando arriviamo a quest'ultima parte dello studio, abbiamo elaborato riflessioni e conclusioni sui partiti politici e sull'attuazione del piano salariale professionale nazionale nei comuni - discorso e pratica. Un percorso considerevole è stato intrapreso alla ricerca della costruzione di un quadro teorico che consentisse non solo di esporre la realtà degli insegnanti in merito all'attuazione della legge $\mathrm{n}$. 11.738/2008, nota come legge nazionale sul salario professionale - PSPN nella sua interezza, in un breve lasso di tempo (2008-2018). Cerchiamo anche di esporre la contraddizione tra i discorsi dei politici che includono negli statuti e nei programmi di partito la difesa del magistero e il contrasto delle loro pratiche nell'esercizio del potere. La contraddizione si configura tra i discorsi magnilosi di apprezzamento dell'insegnante e le pratiche, spesso umilianti.

In nessun periodo storico, come negli ultimi decenni, il ruolo dell'insegnante ha ricevuto tanta enfasi sui dibattiti sui sistemi nazionali e sulle politiche educative e da parte di diversi attori, sia pubblici che privati, e organizzazioni nazionali, internazionali e multilaterali.

La ricerca dimostra la traiettoria di disallineamenti, combattimenti, sconfitte e vittorie che hanno preceduto la discussione e l'approvazione della legge n. 11.738/2008. Allo stesso modo, non sarebbe possibile solo mettere in relazione le parti con il rispetto della legge, senza dimostrare l'intricato sistema politico dei partiti brasiliani, sin dalle sue origini.

La rilevanza di questo lavoro di ricerca è dimostrata per valutare i risultati delle politiche per l'insegnamento e per l'aumento della qualità dell'istruzione, soprattutto nell'ultimo decennio. Vale la pena notare che il Piano Nazionale per l'Educazione ha quattro dei suoi venti obiettivi direttamente correlati alla valorizzazione del magistero. E altri due, che hanno ripercussioni indirette. 
In questa prospettiva, la scelta di questo tema ha rappresentato la possibilità di valutare la gestione pubblica dell'educazione e il confronto di discorsi e pratiche di partito. In questo senso, è stato possibile verificare l'effettiva applicazione della politica di valorizzazione del magistero a partire da uno strumento creato dal MEC con l'obiettivo di mettere a disposizione dei comuni strumenti di informazione, monitoraggio, valutazione e monitoraggio delle politiche per migliorare la qualità dell'istruzione: il Piano di Azioni Articolate - PAR/MEC.

Una prima conclusione è che, in cui così tante discussioni e enfasi sulle politiche di valutazione, annuivano la creazione di una legge che stabilisce il piano salariale per la professione di insegnante, la portata della valutazione effettiva dell'insegnante è lungi dall'essere raggiunta.

L'analisi in 417 comuni dello Stato di Bahia, effettuata ai fini della ricerca finalizzata all'elaborazione della tesi di questo autore[5], ha permesso di raccogliere dati complessivi dal più grande stato del nord-est del Brasile sull'attuazione del Piano Salario del Magistero Baiano, correlandoli ai Partiti Politici davanti ai municipi. Dei 417 comuni censiti, compresi quelli senza partito, solo $146(35 \%)$ rispettano integralmente la Legge $11.738 / 2008$; e dei restanti 271 , sono $30(7,1 \%)$ che non hanno fornito alcuna informazione. Pertanto, si può affermare che 241 comuni $(57,7 \%)$ non rispettano pienamente la legge nazionale sul piano salariale professionale.

Sebbene 180 comuni di Bahia affermino di pagare il valore del PSPN, ciò non significa il pieno rispetto della legge, che ha il suo pieno effetto giuridico solo se articolata al Piano Salario, alla definizione di almeno il 33\% del lavoro come attività oraria $(\mathrm{HA})$ e alla garanzia del piano di carriera. È interessante notare l'esistenza di un singolo comune che dichiara di non avere un piano di carriera, di non rispettare I'HA e di non pagare il PSPN.

Una seconda importante conclusione, e le cifre lo dimostrano, è che solo il $35 \%$ dei comuni bahiano rispetta la legge nazionale sul livello salariale professionale dei 
professionisti dell'istruzione - PSPN, e quasi il $60 \%$, più della metà dei comuni bahiano, non rispetta pienamente la legge 11.738/2008, dopo 10 anni dalla sua sanzione.

Forse, il mancato pagamento del piano giustifica perché la maggior parte degli insegnanti intervistati vuole creare una carriera nazionale per l'insegnamento, lamentandosi di una nuova legge. II risultato della ricerca campionare in 38 comuni ha permesso di confrontarsi e corroborare l'intera indagine documentaria, oltre a portare la realtà di professionisti che lavorano anche in enti sindacali, essenziali nelle lotte per la realizzazione del PSPN. Abbiamo notato, nelle dichiarazioni dei docenti sindacali, pur con alcune differenze, la consapevolezza che una politica di valorizzazione che comprenda il rispetto del piano, condizioni di lavoro adeguate e la formazione iniziale e continua, sarebbe fondamentale per la categoria. Allo stesso modo, la Carriera Nazionale consentirebbe di superare le difficoltà generate dall'autonomia decentrata di cui godono gli enti federali, a causa dell'assenza di un Sistema Nazionale Articolato di Istruzione.

Un'altra importante conclusione della ricerca è che le differenze tra le varie parti non sono significative quando esaminiamo i riferimenti agli insegnanti nei programmi di partito. Dei 32 partiti che includono l'istruzione tra gli argomenti trattati nei loro programmi, solo $12(34,2 \%)$ fanno qualche riferimento agli insegnanti. Alla ricerca di chi cita "valorizzazione, condizioni di lavoro, stipendio e formazione", ci sono 10 sigle partigiane nella seguente distribuzione: 2 a sinistra, 3 al centro e 3 a destra. Ci sono poche differenze importanti tra i partiti di destra, i partiti di centro, i partiti di centrosinistra e di sinistra quando si tratta di discorsi nei programmi di partito e l'effettiva attuazione della legge 11.738/2008. Questo è un risultato inaspettato, dal momento che i partiti di centro-sinistra e di sinistra sono sempre stati in prima linea nelle lotte sindacali e parlamentari nella difesa del Piano Nazionale dello Stipendio Professionale del Magistero.

Nel lavoro di Florestan Fernandes (1979), cerchiamo di capire la ragione di questa piccola differenza importante tra i partiti di destra, centro e sinistra, quando si tratta 
dell'apprezzamento dell'insegnante. In questo caso, per quanto riguarda l'applicazione di politiche permanenti di valorizzazione del magistero e la ragione della mancata applicazione della Legge del Piano Salariale del Magistero da parte dei Partiti Politici, compresi quelli che hanno nei loro programmi la valorizzazione dell'insegnante e ne parlano come un fattore decisivo per la qualità dell'istruzione, il che spiega tante contraddizioni tra i discorsi e le pratiche dei Partiti Politici quando si tratta dell'effettiva valorizzazione dell'insegnante?

Per il sociologo, fondatore della sociologia educativa brasiliana, deputato costituente del Partido dos Trabalhadores Florestan Fernandes, avevamo sull'insegnante un puzzle da decifrare, che consisteva nella tradizione di oggettivazione e brutalizzazione culturale degli insegnanti, propria della cultura brasiliana. Dopo aver citato gli scritti di Marx del 1844, Fernandes aggiunge che in un confronto tra l'insegnante e il proletario, è possibile affermare che l'insegnante era ed è ancora oggettivato nella società brasiliana. Conduit, è un lavoratore intellettuale che non lavora solo con le sue mani. La sfida è, come dice, cercare una comprensione di questa brutalizzazione culturale, che va avanti da così tanto tempo, e che è ancora più pesante quando si tratta di insegnanti che si dedicano all'insegnamento dei bambini, o insegnanti primari (FERNANDES, 1979).

II sociologo afferma, qua e là, che i membri laici e alfabetizzati degli strati sociali dominanti sono entrambi pessimisti sull'efficacia delle istituzioni brasiliane e indifferenti al funzionamento delle scuole e all'insegnamento e al lavoro studentesco (FERNANDES, 1979). Florestan denuncia anche una profonda sfiducia dell'élite nei confronti dell'intellettuale. Per lui, la società brasiliana è fortemente disuguale e molto gerarchica. Se nell'Impero prevaleva la democrazia dei signori, nella Repubblica prevaleva la democrazia degli oligarchi. La democrazia brasiliana è limitata e si rinuncia a una cultura civica, poiché solo una minoranza privilegiata ha accesso alla ricchezza, al potere e alla conoscenza (FERNANDES, 1989).

Quindi, concludiamo che in Brasile, la tradizione culturale e lo scetticismo sembrano essere le cause della svalutazione degli insegnanti e dell'incredulità del potere 
trasformante dell'istruzione. Secondo Florestan, lo scetticismo o il dogmatismo riguardo al ruolo della scuola non significa nulla nelle dinamiche delle trasformazioni sociali. Come rinforzo a queste parole, l'educatore Dermeval Saviani (1987) avverte della necessità che i dirigenti dei movimenti popolari superino la visione della scuola come mero strumento di dominazione borghese, che suscita solo l'interesse della popolazione per la sua capacità di promuovere l'ascensione sociale. A questo punto si può trovare la risposta alla differenza quasi insignificante tra i partiti di destra, centro e sinistra, quando si tratta dell'applicazione del piano salariale dei professionisti dell'istruzione, che non si limita alla retribuzione, ma alla carriera, all'orario di lavoro, alla formazione e alla valorizzazione dell'insegnamento e al conseguente miglioramento della qualità dell'istruzione.

\section{RIFERIMENTI}

BARBOSA, Andreza; FERNANDES, Maria José da Silva Fernandes. O Piso Salarial em São Paulo: desvalorização dos professores. Retratos da Escola, Brasília, DF, v. 10, n. 18, p. 243-257, jan./jun. 2016.

CAMARGO, Rubens Barbosa de; GOUVEIA, Andréa Barbosa; GIL, Juca; MINHOTO, Maria Angélica Pedra. Financiamento da educação e remuneração docente: um começo de conversa em tempos de piso salarial. Revista Brasileira de Política e Administração da Educação - RBPAE, v. 25, n. 2, p. 341-363, mai./ago. 2009.

FERNANDES, Florestan. O dilema educacional brasileiro. In: PEREIRA, Luiz; FORACCHI, Marialice M. (Orgs.). Educação e sociedade: leituras de sociologia da educação. 10. ed. São Paulo: Nacional, 1979.

FERNANDES, Florestan. O dilema educacional. São Paulo: Cortez; Autores Associados, 1989.

FERNANDES, Maria Dilnéia Espíndola; FERNANDES, Solange Jarcem; CAMPOS, Viviane Gregório de. Remuneração docente: efeitos do Plano de Cargos, Carreira e

RC: 101663

Disponibile in: https://www.nucleodoconhecimento.com.br/formazione-it/retribuzionedocenti 
Remuneração em contexto municipal. Ensaio: Avaliação e Políticas Públicas em Educação, Rio de Janeiro, v. 28, n. 106, p. 25-44, jan./mar. 2020.

FERNANDES, Maria Dilnéia Espíndola; RODRIGUEZ, Margarita Victoria. O processo de elaboração da Lei ำ 11.738/2008, Lei do Piso Salarial Profissional Nacional para carreira e remuneração docente: trajetória, disputas e tensões. Revista HISTEDBR On-line, v. 41, p. 88-101, 2011. Disponível em: https://periodicos.sbu.unicamp.br/ojs/index.php/histedbr/article/ view/8639837. Acesso em: 26 set. 2021.

GATTI, Bernardete Angelina; BARRETTO, Elba Siqueira de Sá; ANDRÉ, Marli Eliza Dalmazo de Afonso. Políticas docentes: um estudo da arte. Brasília: Unesco, 2011.

GATTI, Elba Siqueira de Sá Barreto. Professores do Brasil: impasses e desafios. Brasília: Unesco, 2009.

GOUVEIA, Andréa Barbosa Gouveia; FERRAZ, Marcos Alexandre dos Santos. Financiamento da educação e luta sindical: conflitos em uma grande rede de ensino. Educação \& Sociedade, Campinas, v. 37, n. 134, p. 285-302, jan.-mar. 2016. Disponível em: https://www.scielo.br/j/es/a/mvMXV3kDKSmkqbWBq3SvQLL/?lang=pt. Acesso em: 26 set. 2021.

GOUVEIA, Andréa Barbosa; FERNANDES, Maria Dilneia Espíndola. Agenda Sindical de Professores da Educação Básica: desafios postos a partir do Piso Salarial Profissional Nacional. Práxis Educativa, Ponta Grossa, v. 14, n. 1, p. 84-98, jan./abr. 2019. Disponível em: http://www.revistas2.uepg.br/index.php/praxiseducativa. Acesso em: 26 set. 2021.

JACOMINI; Márcia Aparecida; PENNA, Marieta Gouvêa de Oliveira Penna. Carreira docente e valorização do magistério: condições de trabalho e desenvolvimento profissional. Pro-posições, v. 27, n. 2, p. 177-202, maio/ago. 2016. 
LIMA, Maria José Rocha. A trama da ignorância e outros escritos. Salvador: BDA, 1996.

NASCIMENTO, Ana Paula Santiago do; AMORIM, Renata Rodrigues de; CAMARGO, Rubens Barbosa de. Composição salarial dos docentes da Rede Estadual Paulista: uma análise a partir do Boletim de Acompanhamento de Pessoal da SEE/SP, 1996/2010. Fineduca - Revista de Financiamento da Educação, Porto Alegre, v. 4, n. 7, 2014.

OLIVEIRA, Dalila Andrade. As políticas de formação e a crise da profissionalização docente: por onde passa a valorização? Educação em Questão, Natal, v. 46, n. 32, p. 51-74, 2013.

RODRIGUES, Robson da Silva; COSTA, Áurea de Carvalho. Da Constituição Federal de 1988 ao Plano Nacional de Educação 2014-2024: ardilosas apropriações da noção de valorização do trabalho docente. Política \& Trabalho - Revista de Ciências Sociais, n. 50, p. 231-248, jan./jun. 2019.

TEIXEIRA, Eliara Cristina Nogueira da Silva; NUNES, Cláudio Pinto Nunes. O piso salarial como insumo da valorização docente nos governos de FHC e Lula: da Política de Fundos à Lei do Piso. Práxis Educacional, v. 12, p. 251-270, 2016.

\section{APPENDICE - NOTA A PIÈ DI PAGINA DI RIFERIMENTO}

3. Questo articolo fa parte del lavoro di ricerca svolto per la Tesi di Dottorato in Educazione dal titolo Partidos Políticos e Piso Salarial do Magistério Baiano: no Discurso e na Prática, che è nella fase finale di preparazione e sarà difesa presso I'Università Internazionale Iberoamericana (UNINI).

4. Vedi nota a piè di pagina numero uno.

5. Cf. nota n. 1.

Inviato: Ottobre, 2021.

RC: 101663

Disponibile in: https://www.nucleodoconhecimento.com.br/formazione-it/retribuzionedocenti 
Approvato: Novembre 2021.

RC: 101663

Disponibile in: https://www.nucleodoconhecimento.com.br/formazione-it/retribuzionedocenti 